\title{
Quantum Dot Systems: a versatile platform for quantum simulations
}

\author{
Pierre Barthelemy* and Lieven M. K. Vandersypen
}

Received 7 June 2013, revised 8 August 2013, accepted 14 August 2013

Published online 18 September 2013

Quantum mechanics often results in extremely complex phenomena, especially when the quantum system under consideration is composed of many interacting particles. The states of these many-body systems live in a space so large that classical numerical calculations cannot compute them. Quantum simulations can be used to overcome this problem: complex quantum problems can be solved by studying experimentally an artificial quantum system operated to simulate the desired hamiltonian.

Quantum dot systems have shown to be widely tunable quantum systems, that can be efficiently controlled electrically. This tunability and the versatility of their design makes them very promising quantum simulators. This paper reviews the progress towards digital quantum simulations with individually controlled quantum dots, as well as the analog quantum simulations that have been performed with these systems. The possibility to use large arrays of quantum dots to simulate the low-temperature Hubbard model is also discussed. The main issues along that path are presented and new ideas to overcome them are proposed.

\section{Introduction}

Simulating quantum many-body physics on a classical system is a tremendous task. The complexity of calculations increases exponentially with the number of particles and strongly limits the size of the systems that can be described. To overcome this limitation, Feynman [1] introduced the notion of quantum simulations: to create an artificial controllable quantum system and use it to simulate a complex many-body phenomenon. Questions that could be answered efficiently with such a quantum simulator include the nature of the ground state of a given Hamiltonian, the energy spectrum, the time dynamics and so forth.
The promises of quantum simulations caused a lot of excitement, especially when cold atoms systems allowed controlling interparticle interactions and simulating strongly correlated quantum systems [2]. Interesting simulated problems include metal-insulator transitions $[3,4]$, the onset of magnetism [5], or relaxation in isolated systems [6], for which cold atoms could simulate the dynamics for a much longer time span than any classical algorithm. Recently, trapped ions [7-9] allowed to simulate spin-spin interactions and to study frustrated spins systems. Moreover, they also allowed to implement digital quantum simulations [10] and open the way towards a universal quantum simulator [11]. Nowadays, analog and digital quantum simulations can be performed using cold atoms [2, 12], trapped ions [9, 13], photonic [14, 15] or superconducting circuits $[16,17]$.

Quantum dot systems [18-20] have a very interesting role to play in this field. They confine single electrons in a series of sites, where the coupling between sites, between sites and external electron reservoirs and the electrochemical potential of a site can be widely and precisely tuned electrically. At low temperature $(T \approx 10-$ $100 \mathrm{mK}$ ), inter-particle interactions and spin interactions as well as their interplay are all sufficiently strong to be observed. Such an interplay is generally difficult to observe in other quantum simulators (except at the local scale in bichromatic optical lattices [21]).

Electrons in quantum dots are therefore very promising candidates for quantum simulations. They not only naturally obey the Hubbard hamiltonian, but typical parameter values [18-20] of charging energy $(U \approx$ $1 \mathrm{meV})$, interdot tunnel coupling $(t \approx 10-100 \mu \mathrm{eV})$ and dilution refrigerator temperature $(k T \approx 1-10 \mu \mathrm{eV})$ give access to one of the most interesting regimes of the Hubbard hamiltonian. Specifically, the relation

\footnotetext{
* Corresponding author E-mail: p.j.c.barthelemy@)tudelft.nl Kavli Institute of Nanoscience, TU Delft, 2600 GA Delft, The Netherlands
} 
$k T \ll t \ll U$ gives rise to very complex quantum phase diagrams, where spin order and charge order compete and give rise to magnetic phases, pseudogap phase, stripe states and possibly superconductivity $[22,23]$. This low-temperature regime has been so far very difficult to study theoretically [24] or through other quantum simulators [25].

As we will see, it is also possible, by a dedicated sample design or by a careful tuning of the dot parameters, to force the system to simulate other Hamiltonians, such as Heisenberg, Ising or Kondo Hamiltonians.

This paper aims to give an overview of the quantum simulations performed to date with quantum dots, and to present proposals for further developments. It will be structured as follows: in section 2, we will describe the physics of gate-defined quantum dots. We will see how one can define a series of coupled quantum dots to trap single electrons and we will review the main experiments using these dots for both digital and analog quantum simulations. In section 3 we will review the physics of large arrays of quantum dots. Starting from the early developments, we will show the progress in this field towards simulating correlated electron physics in large scale systems. In section 4 , we will present new ideas to overcome the main issue this field is facing: the relatively large impurity-induced disorder in semiconductor systems. Sections 3 and 4 can be read independently of section 2 .

\section{Few quantum dot systems}

\subsection{Quantum Dots: Localized charge and spin}

Quantum dots are man-made structures designed to trap electrons on length scales varying from a few tens to a few hundreds of nanometers. These dots can be capacitively coupled to electrostatic gates that are used to modify their equilibrium charge state, and can be tunnel coupled to electron reservoirs or to neighbouring quantum dots $[18,19]$. In this review, we will focus on gate-defined dots, as their intrinsic electrical tunability makes them particularly attractive for quantum simulations.

Gate-defined quantum dots are usually based on semiconductor heterostructures presenting a quantum well in the vicinity $(\sim 50-100 \mathrm{~nm})$ of the surface. Different materials can be used, such as GaAs/AlGaAs [19] or $\mathrm{Si} / \mathrm{SiGe}$ [27]. Figure 1a shows a typical heterostructure on which gate-defined quantum dots are built: it consists of an AlGaAs/GaAs heterojunction, grown by molecular beam epitaxy. The difference in bandgap energy for the two materials results in a step in the conduction band
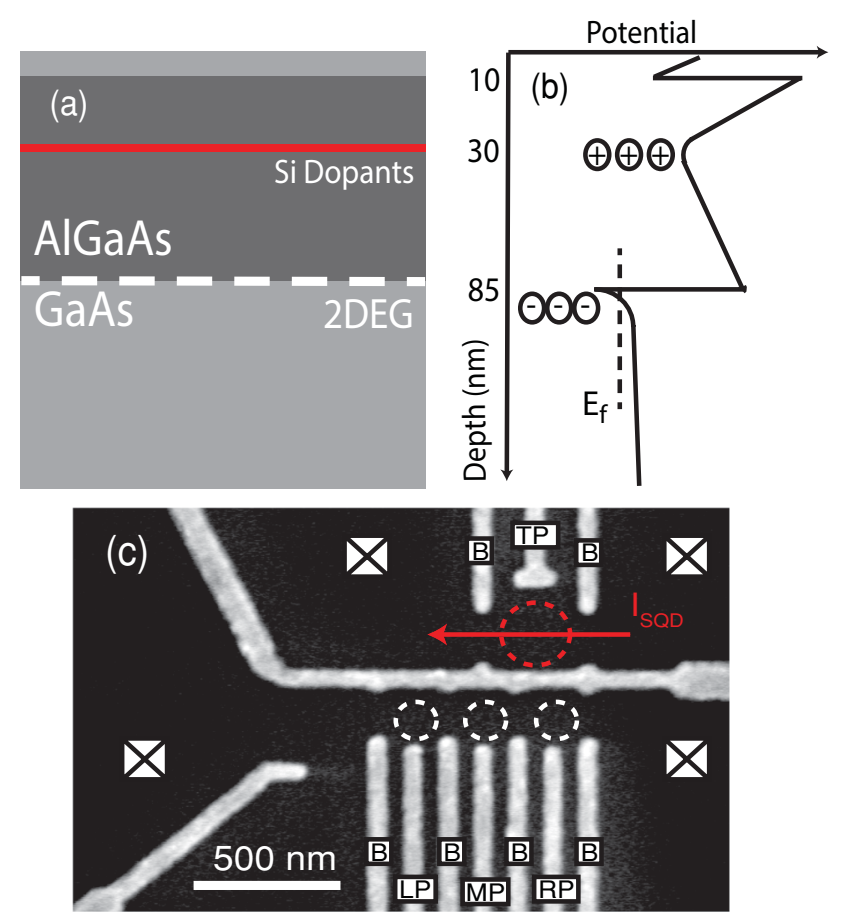

Figure 1 Gate defined quantum dots. (a) Typical heterostructure design: the 2DEG is formed at the GaAs-AlGaAs heterointerface. (b) Conduction band diagram: the presence of dopants creates localized positive charge that pulls the conduction band minimum below the Fermi energy $E_{f}$ at the heterointerface, which confines the electrons in a 2D electron gas (2DEG). (c) SEM image of a triple dot device (white dashed circles in the lower half) capacitively coupled to a large single dot (red dashed circle in the upper half). (From Ref. [26])

minimum at the interface. During the growth process, a thin Si-doped layer is placed above the heterojunction. As shown in the conduction band diagram of Fig. 1b, the ionized dopants locally bend the conduction band minimum. This effect creates a triangular quantum well at the interface in which electrons are bound, forming a twodimensional electron gas (2DEG).

Using electron-beam lithography, one defines thin metallic gates on the surface of the semiconductor $[19,20]$. Figure 1c shows a scanning electron microscope (SEM) image of the gates used to define a triple dot system (bottom half) capacitively coupled to a single dot (upper half). Applying a negative voltage on the gates creates potential wells surrounded by barriers tunable by the "B" gates, in which a certain number of electrons is trapped. Applying a negative voltage on the plunger gates ("LP", "MP", "RP" and "TP") lowers the number of trapped electrons in each well or quantum dot down to the single-electron regime. The crossed squares in 
Figure 1c indicate electron reservoirs, which are connected to external electronics through ohmic contacts.

Gate-defined quantum dots can also be created in nanowires [28] or carbon nanotubes [29]. We will focus in this review on 2DEG-based devices, as the versatility of the $2 \mathrm{D}$ design is especially interesting for quantum simulations.

The 3D confinement of electrons in the quantum dot creates a discrete series of quantum states. Due to the huge difference in the amplitude of the in-plane confining potential (defined by the gates) and of the confinement in the growth direction (due to the heterostructure), the low-energy states depend only on the 2D lateral potential, and display therefore a 2D shell structure. Quantum dots are therefore often termed "artificial 2D atoms". As the filling of these energy levels is determined by Hund's rule, one can build a periodic table for $2 \mathrm{D}$ elements [18].

An important quantity that controls the behavior of electrons in the dot is the electrochemical potential for the $\mathrm{N}$-electron state, $\mu(N)$, defined as the energy needed to add the $N$ th electron to a $N-1$ electron dot:

$\mu(N)=E(N)-E(N-1)$,

where $E(N)$ is the energy of the N-electron state. Electrochemical potentials of successive charge states are spaced by the addition energy:

$\mu(N+1)-\mu(N)=E_{\text {add }}(N)=E_{C}+\Delta E$,

where $E_{C}$ is an electrostatic charging energy, determined by the coulomb interaction of the $N$ th electron with other electrons inside and outside the dot, and $\Delta E$ the quantized energy level spacing in the artificial atom. In small dots $(\sim 50 \mathrm{~nm})$, typical charging energies are of the order of a few $\mathrm{meV}$, and energy level spacings are of the order of $1 \mathrm{meV}$. The electrochemical potentials corresponding to different electron numbers form therefore a ladder with steps separated by a few $\mathrm{meV}$. As the dots are capacitively coupled to the gates, $\mu(N)$ depends linearly on gate voltages. Thus varying a gate voltage shifts the whole ladder up and down, without modifying the step spacing.

Current flows through a quantum dot via a succession of charge transitions: starting from a N-electron state, one electron exits to the right lead, while another electron enters from the left lead (see Fig. 2a). This can occur only if the electrochemical potential $\mu(N)$ lies within the bias window defined by the electrochemical potentials of the two electron reservoirs: $\mu_{D} \leqslant \mu(N) \leqslant \mu_{S}$. Transport then occurs via the charge transitions $N \rightarrow N-1 \rightarrow N$.

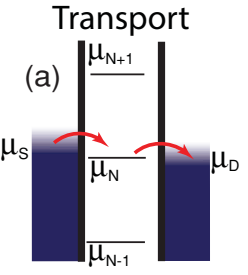

Coulomb Blockade
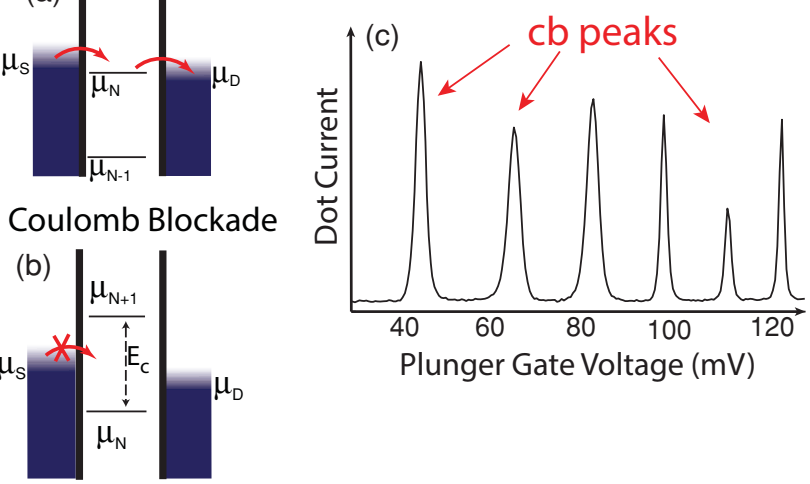

Figure 2 Coulomb Blockade. (a) When the electrochemical potential of the $\mathrm{N}$-electron state lies in the bias window for some $N$, current can flow through the dot. (b) When no electrochemical potential levels lie in the bias window, current is blockaded. (c) Current through the single dot of Fig. $1 \mathrm{c}$ as a function of the plunger gate voltage. A Coulomb-blockade (cb) peak appears each time a level enters the bias window.

When there is no electrochemical potential level inside the bias window, direct tunneling of electrons cannot occur and transport is blockaded (Fig. 2b). This phenomenon is called Coulomb Blockade. Figure 2c shows the current trough the single quantum dot of Fig. 1(c), displaying Coulomb blockade peaks: varying the potential of the plunger gate shifts the whole electrochemical potential ladder and results in a current peak each time one of the electrochemical potentials is aligned within the bias window. In the valley, the residual current is determined by higher-order processes such as cotunneling: electrons go from one lead to the other virtually occupying higher energy levels.

If a single quantum dot behaves like an artificial atom, quantum dots placed next to each other can form "artificial molecules" [19, 30, 31]. For the device shown in Figure 1c a triple dot array is formed in the lower half of the image. The voltage on the "B" gates controls the interdot tunnel barriers, and the voltage on the plunger gates ("LP", "MP" and "RP"), allows to shift the electrochemical potential ladders of the different dots individually. The interdot tunnel coupling hybridizes the charge states localized on each of the respective dots, thereby forming "bonding" and "antibonding" orbitals. This effect can be used to perform quantum simulations of chemical reactions [32].

The current through a quantum dot system as a function of the various gate voltages and the bias voltage can be used to infer the number of electrons occupying each 


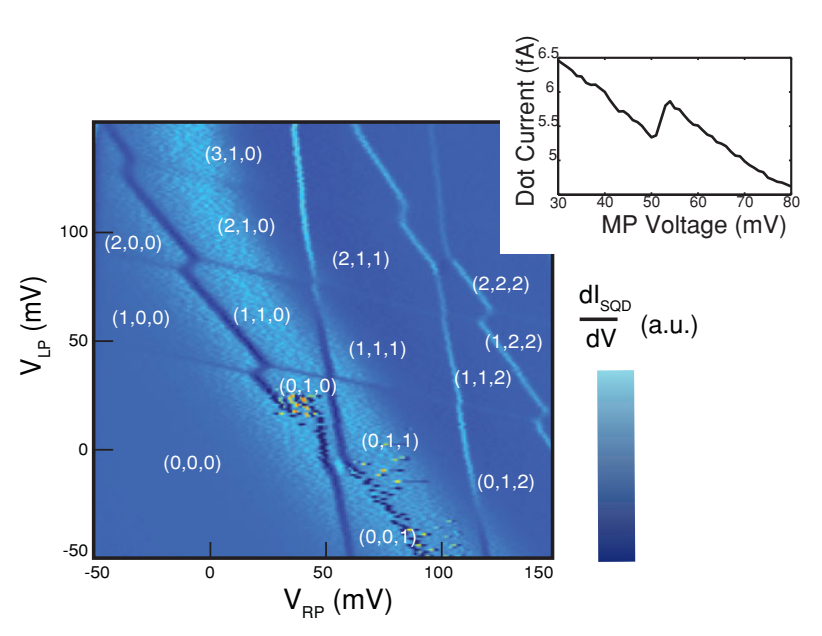

Figure 3 Charge sensing in a triple dot (from Ref. [26]. Tuning the sensing dot in Figure 1c to the flank of a Coulomb Blockade peak gives a conductance that is strongly sensitive to changes in the electrostatic environment. (inset) Modifications of the charge state of a nearby quantum dot results in measurable current steps. (main figure) Differential conductance measurements as a function of two plunger gate voltages allows to draw charge stability diagrams, where modifications of the charge state of different dots occur at lines whose slope is determined by dot-gates capacitive couplings. Stable charge states are noted $\left(N_{L}, N_{M}, N_{R}\right)$, with $N_{L}, N_{M}, N_{R}$ being the number of electrons in the left, middle and right dot.

dot, the orbital level spacing, spin splittings, and so forth. A development of the last ten years is that all this information can also be obtained via a charge sensor placed in the vicinity of the quantum dots. Such a sensor can be easily integrated in the form of a narrow channel (quantum point contact or QPC) [33] or another dot [34]. The sensor must be operated at a working point where its conductance is sensitive to the electrostatic environment (i.e. on a flank of a Coulomb peak). Changes in the occupation of any of the dots are easily detected using such sensors (see Figure 3). We also see in Figure 3 that by adjusting the gate voltages, any desired number of electrons can be placed on any of the dots.

As we have seen, gate defined quantum dots offer a quantum platform with very high controllability and very efficient charge readout schemes. We will see in the next section how this can be used for digital and analog quantum simulations.

\subsection{Towards digital quantum simulation}

The principle of digital quantum simulations $[1,10,37]$ is to program a quantum computer for simulating the dy- namics of a certain Hamiltonian $H$. The simulated initial state is represented by the quantum state $\psi_{0}$ of a qubit register, and the time-evolution operator is approximated by a succession of elementary quantum gate operations acting on this qubit register.

Practically, if $H$ is local, it can be decomposed in a sum of terms $H_{k}$ acting on a finite number of particles. The time evolution due to $H_{k}$ can be represented by a series of gate operations on the qubit register. However, as all the $H_{k}$ may not commute, the product of the evolution operators $U_{k}(t)=e^{-i H_{k} t / \hbar}$ is not equal to the total time evolution $U(t)=e^{-i H t / \hbar}$. One can solve this by using the Trotter approximation:

$$
U(t)=\lim _{n \rightarrow \infty}\left(\prod_{k} U_{k}\left(\frac{t}{n}\right)\right)^{n} .
$$

This means that discretizing time in small intervals and performing, for each time interval, a succession of quantum gate operations on the qubit register, on can approximate the time evolution due to any local Hamiltonian. The requirements for implementing digital quantum simulations are thus the same as for a universal quantum computer [38].

Quantum dots can be used as qubits in various ways. The most promising approaches rely on spin manipulation in quantum dots in the few-electron regime. Qubit implementations based on charge degrees of freedom suffer from very short coherence and relaxation times ( $T_{2}<10 \mathrm{~ns}, T_{1} \approx 10 \mathrm{~ns}$ ) [39-41].

When a quantum dot is filled with a single electron, the spin-1/2 of this single electron becomes a localized magnetic moment. Applying an in-plane magnetic field $B$ (defining the $z$ direction) lifts the spin-degeneracy of the electronic states by the Zeeman energy: $\Delta E_{Z}=$ $g \mu_{B} B$, with $\mu_{B}$ Bohr's magneton, and $g$ the g-factor, which depends on the heterostructure material and on the dot shape and dimensions. One defines Zeeman qubit states by the projection of the spin on the magnetic field axis: $|0\rangle=|\uparrow\rangle$ and $|1\rangle=|\downarrow\rangle$ [42]. Note that as GaAs has a negative g-factor $(g \approx-0.44)$, the ground state $|0\rangle$ is a spin-up state. The magnetic field is typically a few Tesla, so that the Zeeman energy exceeds significantly the thermal energy.

Single spins can be manipulated by electron spin resonance [43] (ESR): an oscillatory current applied to a strip line placed next to a dot, induces an oscillatory magnetic field in a direction perpendicular to the magnetic field, which rotates the electron spin when it is on resonance with the Zeeman splitting. Alternatively, one can use electric-dipole spin resonance (EDSR) [35, 44], which allows all-electrical control of the spin: applying 

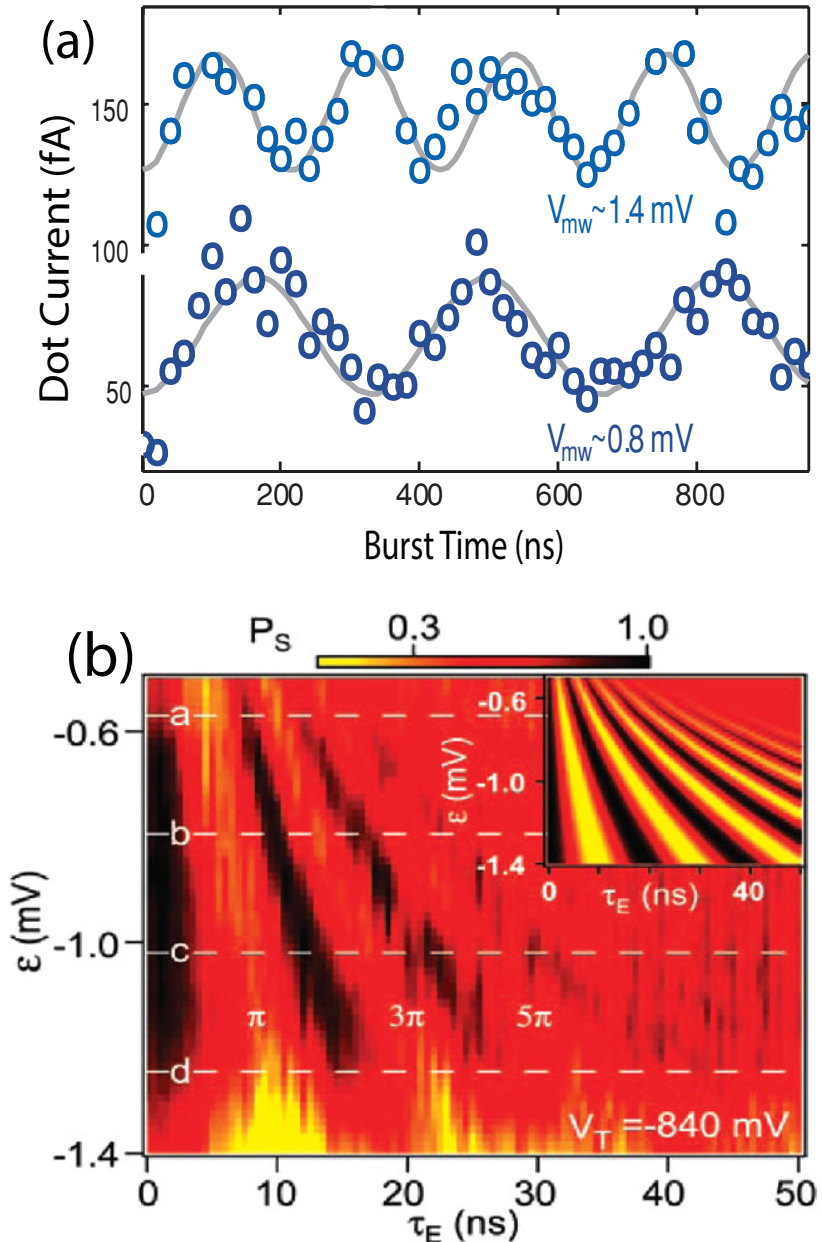

Figure 4 Electron spin manipulation in quantum dots. (a) EDSR forces spin rotations in one of the dots. The rotation angle depends on the pulse duration. This rotation, through Pauli Spin Blockade, results in a variation of the current through a double dot (from Ref. [35]). (b) Pulsing the detuning induces spin rotations between singlet and triplets: the probability $P_{s}$ of measuring a singlet state in a double quantum dot shows oscillations depending on the duration $\tau_{E}$ and the detuning $\epsilon$ of the pulse (from Ref. [36]).

ac excitation on the electrostatic gates forces the quantum dot position to oscillate. Due to the spin-orbit coupling in the semiconductor, the motion of the electrons results in an effective oscillating magnetic field that rotates the spins with Rabi frequencies in the Megahertz range. EDSR can also be performed using a local micromagnet to create a magnetic field gradient. The motion of the electrons in the inhomogeneous magnetic field also allows controlled spin rotations [45].

Due to the combined effect of Pauli exclusion principle and Coulomb interactions, single electrons in two neighbouring dots feel an effective spin-spin interaction. This interaction takes the form of an Heisenberg ex- change coupling [42]:

$H=-J \mathbf{S}_{1} \cdot \mathbf{S}_{2}$,

where $S_{i}$ is the spin of the electron in dot $i$. The coupling constant $\mathrm{J}$ is negative (antiferromagnetic coupling) and depends on the spatial overlap of the electron wave functions, which is controlled by gate voltage. This coupling is the basis of a two-qubit gate for Zeeman qubits [42]. The effect of the exchange interaction is literally to swap or exchange the state of the two spins [46]. When exchange acts for half the duration of a SWAP gate, the $\sqrt{S W A P}$ gate is obtained, which is universal when combined with single-spin rotations. Exchange gates can be very fast, and are in practice limited only by the achievable rise times of gate voltage pulses (sub-ns).

An alternative qubit representation based on spins in quantum dots uses two-electron singlet and triplet spin states in a double quantum dot to define a single qubit [47]. The two-spin exchange interaction now acts as a single qubit gate [36]. For such singlet-triplet (ST) qubits a two-qubit gate requires to couple two double dots. Capacitive coupling between the dots can be used here: the difference in charge configuration between singlet and triplet state in one double dot slightly modifies the singlet-triplet time evolution in the other, which allows to entangle the spin states of the two double dots [48]. More recently, also a triple quantum dot containing three spins was operated as a single qubit [49-52], using the spin exchange mechanism to drive single qubit operations. Each of the possible qubit representations has its own advantages and disadvantages, and the improvement of the spin qubit performances is an actively pursued research topic.

To read out spin qubit states, two methods have been successfully demonstrated (see Figure 5). In both cases, tunneling of electrons is made to depend on their spin states. Simultaneous detection of the charge occupation of the dots, then allows one to infer the spin states. For single spins, the energy difference between spin-up and spin-down electrons is exploited to induce spin-selective tunneling (see Figure 5a) [53]. For two-spin states, the so-called Pauli spin blockade technique is used (see Figure 5b) $[54,55]$.

Relaxation of spins in quantum dots typically occurs via phonon-emission $[20,56]$. Coupling of spins to phonons is usually dominated by the spin-orbit interaction (SOI), which admixes spin and orbital states, making spins sensitive to electric field fluctuations. Spin relaxation times strongly depend on the spin splitting, as this affects the relevant phonon density of states and electron-phonon coupling [57]. For low magnetic fields, 

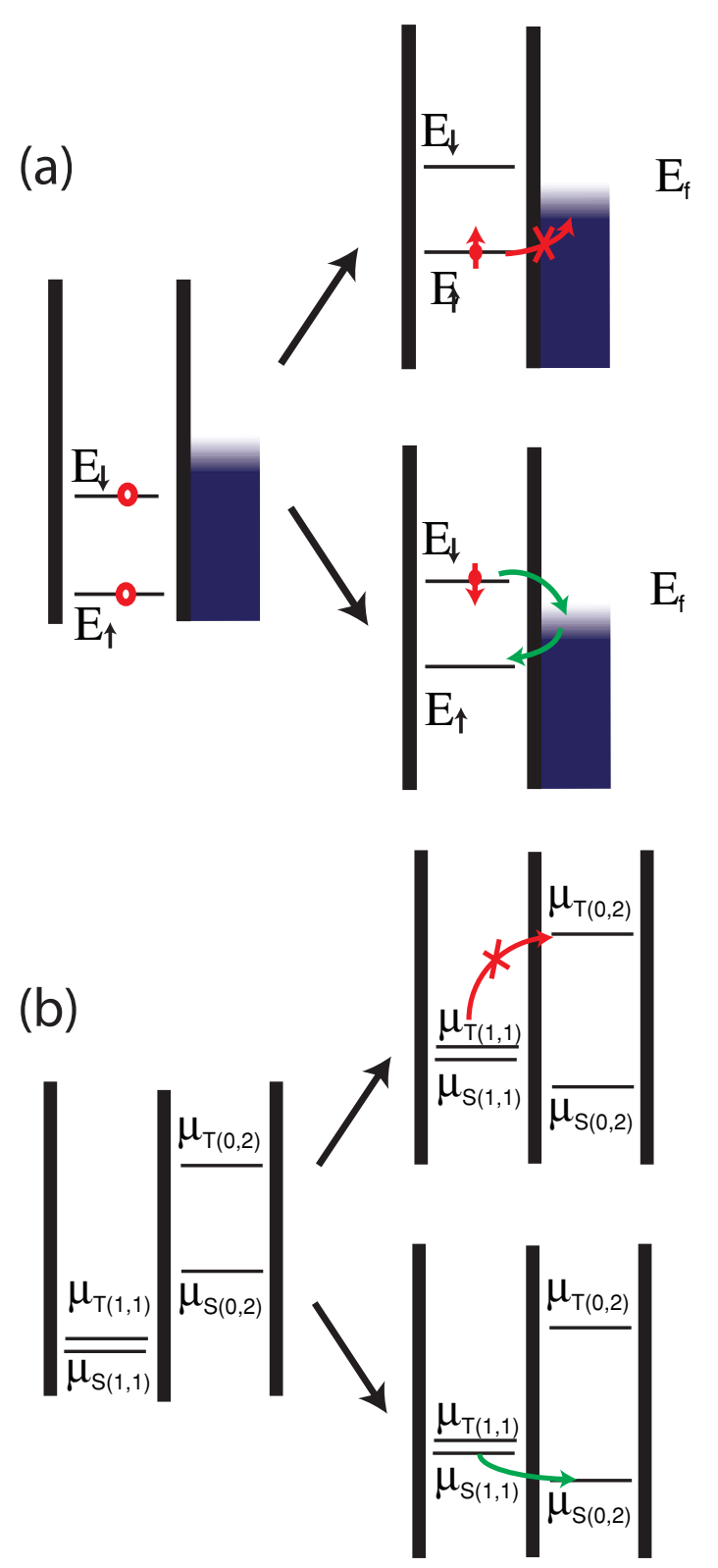

Figure 5 Single Shot Readout Methods. (a) Readout for Zeeman qubits [53]: initially, both spin-up and spin-down states are below the Fermi level of the lead. Pulsing the plunger gate potential to a configuration where only the spin-down state is above the Fermi level induces spin-to-charge conversion: only spin-down can tunnel out of the dot. Therefore, measuring in real time the variations of the charge state allows to infer the spin state of the electron. (b) Pauli Spin Blockade Readout for ST qubits [54,55]: initially, the double dot is in the $(1,1)$ charge state for any spin configuration. Pulsing the detuning, only a singlet state can reach the $(0,2)$ charge configuration. For triplet states, tunneling to $(0,2)$ is forbidden by the high singlet-triplet splitting in the $(0,2)$ configuration. Monitoring the charge configuration permits to read out the spin: measuring a $(0,2)$ charge state implies that the qubit is in a singlet state, while measuring a $(1,1)$ charge state points at a triplet state. relaxation times as high as $T_{1}=1 \mathrm{~s}$ have been reached, both in GaAs [58] and in Si quantum dots [59].

The spin-orbit coupling does not induce pure dephasing of the spin-state, so it only influences spin coherence through relaxation and sets a limit $T_{2}<2 T_{1}$ [60]. Unfortunately, other perturbations strongly influence the phase of the spin states. The main source of spin decoherence is the hyperfine coupling between the electron spin and the spin of the Ga and As nuclei forming the quantum dots $[20,61,62]$. Interestingly, this hyperfineinduced decoherence in quantum dots is a physical realization of the well-known Central Spin problem, a heavily studied problem in condensed matter physics that in general cannot be solved exactly by analytical or numerical methods. The basic question is how the coherence evolves for one central spin that is coupled to a bath of other spins [63]:

$$
H_{\text {hyp }}=\sum_{i} A_{i} \mathbf{S}_{i} \cdot \mathbf{S},
$$

where $\mathbf{S}_{\mathbf{i}}$ is the spin of the $i^{\text {th }}$ nuclei and $A_{i}$ the coupling strength, proportional to the value of the electron wavefunction at the position of the $i^{\text {th }}$ nuclei. For large numbers of nuclei (for GaAs quantum dots, $N_{\text {nuclei }} \approx 10^{4}-$ $\left.10^{6}[20]\right)$, the influence of the nuclei can be modelled semiclassically as an Overhauser magnetic field $B_{n u c}$, and the hyperfine interaction can be described by

$$
H_{h y p}=g \mu_{B} \mathbf{B}_{n u c} \mathbf{S}
$$

In strongly polarized systems, $B_{n u c}$ can be as high as a few Tesla, while its statistical fluctuations are of the order of a few $\mathrm{mT}[20,61,62]$. This effective magnetic field adds up to the applied magnetic field and dephases the spins with characteristic time $T_{2}^{*} \approx 10-100 \mathrm{~ns}[36,43]$. Using spinecho pulses, this dephasing can be compensated for. The resulting decoherence time is limited by nuclear spin dynamics: $T_{2} \approx 1-100 \mu \mathrm{s}[36,64,65]$.

Intense efforts focus nowadays on improved performance of coherence and control of spins. As the most important limitation comes from the hyperfine coupling, nuclear spin-free systems would be highly desirable. $\mathrm{Si} / \mathrm{SiGe}$ heterostructures offer very interesting possibilities in this domain: they have a very low concentration of nuclear spins [27], and can be isotopically purified [66]. The improvements due to the weak hyperfine coupling in $\mathrm{Si} / \mathrm{SiGe}$ systems are seen in a measured singlet-triplet dephasing time of $T_{2}^{*}=360 \mathrm{~ns}$ [67], which is up to 40 times longer than the typical case in GaAs [36]. Furthermore, singlet-triplet relaxation times as high as 3s have been measured [68], compared to at most $10 \mathrm{~ms}$ in GaAs systems [69]. 
Another option is to control the nuclei and stabilize the Overhauser field by electron spin-nuclear spin feedback [61,62]. Using these methods, a $T_{2}^{*}$ increase of a factor of 10 has been demonstrated in GaAs [70].

These ideas could lead to important improvements of the performance of quantum dot spin qubits and could open the way towards digital quantum simulations with quantum dots.

\subsection{Analog Quantum Simulations}

Whereas using quantum computers should lead to a universal quantum simulator, analog quantum simulations can be performed by constructing dedicated quantum systems designed to simulate a specific Hamiltonian. It is of course not sufficient for a quantum system to evolve according to a Hamiltonian to qualify as a quantum simulator. A system becomes a useful simulator when it has control knobs that allow to freely tune the relevant parameters of the Hamiltonian, or when it permits measurement of important quantities that are not accessible in natural systems. In this way, the Hamiltonian can be taken into regimes that were not accessible before, and that are too complex to be studied via classical computations, giving new insight in the underlying physics.

Quantum simulators are especially interesting to study the emergence of strongly correlated phases in many-body systems, as these regimes are the most difficult to study theoretically or simulate classically. For this purpose, quantum dots show very interesting properties: as we have seen in the previous section, both charge and spin correlations are present in quantum dots, and can present a complex interplay already in double or triple dot systems.

An array of tunnel coupled quantum dots is naturally described by the Hubbard hamiltonian [71] and all the parameters of the hamiltonian (interdot detuning, tunnel couplings, ...) can be widely varied by controlling the gate voltages. Quantum dot systems can therefore be used to simulate Hubbard physics. The form of this hamiltonian is

$$
H=-\sum_{i, j} t_{i, j} c_{i}^{\dagger} c_{j}+\sum_{i} U_{i} n_{i, \uparrow} n_{i, \downarrow}+\sum_{i} \mu_{i} n_{i}
$$

The first term describes the tunnel coupling between dot $i$ and dot $j, U_{i}$ is the on-site interaction energy in dot $i$, and the $\mu_{i}$ is the chemical potential of electrons in dot $i$, which depends linearly on gate voltages. Note that this hamiltonian is a single band hamiltonian that takes into account only one orbital state per dot. As explained above, in typical structures, $U$ is of the order of a few $\mathrm{meV}$, $t$ is tunable by gate voltages from $t \simeq 0$ to $t \sim 100 \mu \mathrm{eV}$ and all $\mu_{i}$ can be controlled individually over a range of several meV. In a dilution refrigerator, such a system can be cooled down to a few tens of $\mathrm{mK}$, ensuring that the thermal energy is significantly lower than the other energy scales in the system $(k T \ll t \ll U)$. This regime is particularly important for condensed matter, yet is hard to access using cold atoms in optical lattices. Therefore quantum dots offer a very interesting platform for simulation of the Hubbard hamiltonian.

The few-quantum dot systems recently studied experimentally $[26,48,72-74]$ show an increasing complexity. They include triple dots in either a linear or triangular geometry, and quadruple dots arranged along a line or a square. In long chains ( $\geq 4$ dots), 1D correlated electron physics [75] could be simulated. Two-dimensional arrays of dots $(\geq 3 \times 2)$ could lead to the simulation of Hubbard ladder physics [76], and the observation of d-wave hole pairing and possibly superconductivity [77].

The currently available systems already present very interesting possibilities: for instance, using a fully tunnel-coupled quadruple dot with three electrons, one could observe Nagaoka's ferromagnetism [78-80], which takes its origin in a two-path interference process (see Figure 6). Let us first assume infinite interactions ( $U=$ $\infty)$, which totally prevent double occupancies. The four dots are denoted by the index $i=1,2,3,4$, and neighbouring dots are tunnel coupled. Without double occupancies, the charge state of the three-electron system is completely characterized by the position of the hole $(|2>=|(1,0,1,1)>)$. The origin of Nagaoka's ferromagnetism is found in the matrix elements $<1|H| 3>$ and $<2|H| 4>$, describing the coupling between nextnearest neighbour sites on the quadruple dot plaquette. For aligned spins (ferromagnetic phase), the two paths $|1>\rightarrow| 2>\rightarrow \mid 3>$ and $|1>\rightarrow| 4>\rightarrow \mid 3>$ are indistinguishable and their contributions to $<1|H| 3>$ interfere constructively. In contrast, the two paths are distinguishable for non-aligned spins, and therefore their contributions do not interfere (see Fig. 6a-b). Due to this effect, $|<1| H|3>|$ is higher in the ferromagnetic case, which means the hole can lower its kinetic energy by delocalizing. This makes the ferromagnetic state the ground state for large $U$. For negligible $U$, double occupancy of any site is allowed. Now the ground state has two electrons in the lowest energy (delocalized) orbital (forming a singlet) and one electron in the next orbital. The ferromagnetic (spin-3/2) state can have only one electron in each orbital, giving a larger overall energy. This destroys the ferromagnetic phase for $U / t<18.6$ (see Figure 6d). The gap between the two spin configurations for $U=1 \mathrm{meV}$ is shown in Fig. 6e. We see that for realistic values of $t$ in a 


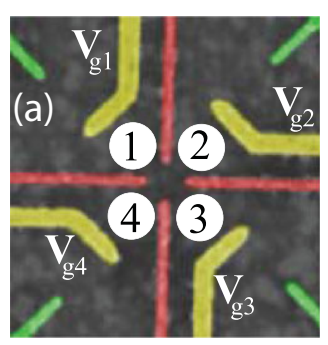

(b)
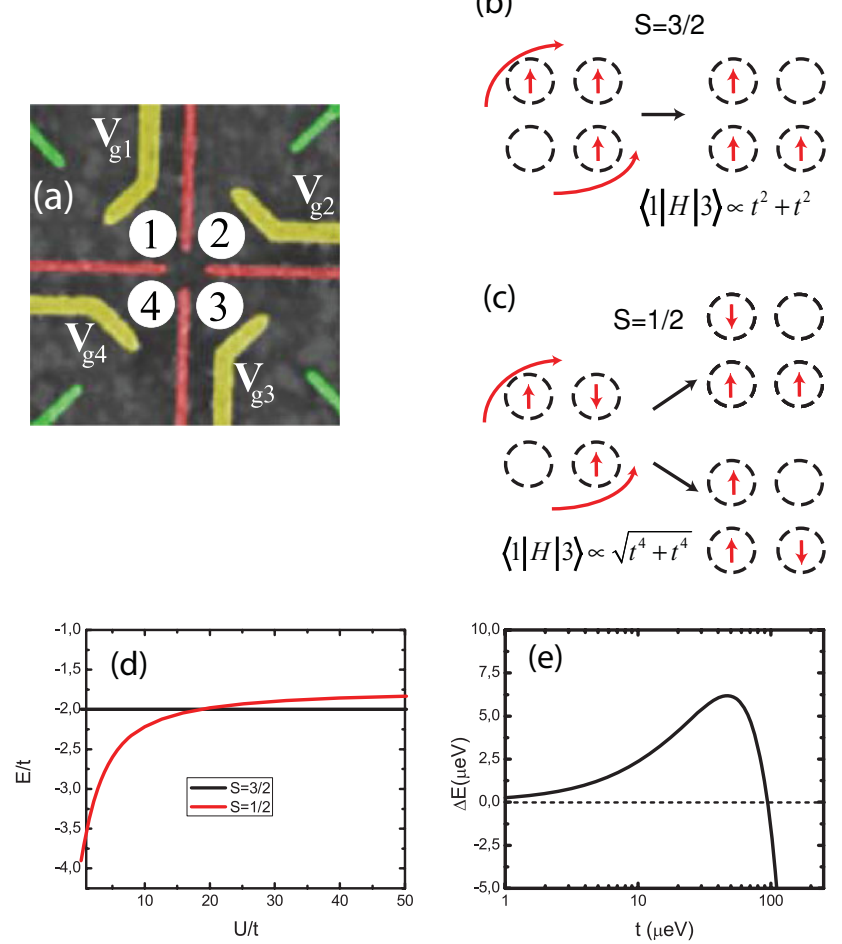

Figure 6 Nagaoka's ferromagnetism in a quadruple dot (a) SEM image of a ring-coupled quadruple dot system. ( from Ref. [74]. (b) For the spin $3 / 2$ (ferromagnetic) case, two paths add up constructively to contribute to $<1|H| 3>$ (c) For spin $1 / 2$, these two paths are distinguishible and do not interfere. (d) Energy diagram as a function of $U / t$. One can see that the ferromagnetic $(\mathrm{S}=3 / 2)$ state is the ground state for $U / t>18.6$. (e) The gap between the $S=3 / 2$ and $S=1 / 2$ states can be as high as $7.5 \mu \mathrm{eV}$

quantum dot array, this gap should be observable, as well as the quantum phase transition between the ferromagnetic and unpolarized states.

Another recent development could have interesting applications for quantum simulations: in a triple-dot system, long-distance charge coupling (due to cotunneling) has been recently demonstrated [26]. This could lead to new schemes of spin-spin coupling between non-nearest neighbours and could allow simulating frustrated spin systems combining exchange coupling between nearest neighbours with non-local coupling as the frustrating link.

The flexibility of the design of quantum dots constitutes one of their major advantages: indeed, complex geometries can be built, allowing the study of certain Hamiltonians in very different situations, bringing new insight into interesting quantum effects. In that respect, the study of the Kondo effect in quantum dots gives a very interesting illustration of the versatility of quantum dots systems.
The Kondo effect [81] was first observed in metals doped with magnetic impurities. These systems show a resistivity that, below a certain critical temperature, increased upon decreasing temperature. This effect is the consequence of the coupling between the spin of the free conduction electrons with the localized magnetic moments on the impurities. This spin coupling forces the localized and delocalized electrons to form a spin singlet, which increases the density of states at the Fermi energy. This increased density of state favors scattering and increases the resistance in metallic system.

The Kondo hamiltonian can be derived from the Anderson impurity model [82] describing electrons bound to localized states inside a metal host, which explains the origin of the spin interaction as cotunneling (secondorder tunneling) of conduction-band electrons on the impurity. A quantum dot with an odd number of electrons coupled to electron reservoirs is a powerful quantum simulator for the Anderson impurity model. Indeed, conduction can take place in a Coulomb-blockade valley due to cotunneling [83], which couples the dot electron spin to the density of spins in the leads, giving rise to an increased density of states at the Fermi energy. This peak in the density of states can be accessed through differential conductance measurements [84, 85]: $d I / d V$ curves show a zero-bias peak whose height increases upon decreasing temperature (see Figure 7a). Note that the coupling between localized and conduction spins manifests itself through an increasing resistance in metals and through an increasing conductance in quantum dots.

The tunability of the parameters of the quantum dot also allowed to study spin-1 Kondo physics in an evenlyoccupied quantum dot [86]. Furthermore, as we discuss next, the versatility of the design of quantum dot systems allowed simulating more complex hamiltonians, giving insight into multichannel Kondo coupling or multi-impurity Kondo effects.

For impurities coupled to two reservoirs independent from each other (electrons cannot be exchanged between them), a two-channel Kondo effect can occur if the coupling to both reservoirs has the same strength: instead of building a singlet with one of the reservoirs, the spin of the electron in the dot induces correlations between the two reservoirs. This effect is relevant for understanding heavy fermions $[87,88]$ and glassy metal [89] physics. To study it, Potok et al. [90] built a quantum dot coupled to both a finite (Fig. 7e, red colored) and an infinite reservoir (Fig. 7e, blue colored). Due to its finite size, the red reservoir has a finite charging energy, and electrons cannot be exchanged between red and blue reservoirs. Varying the voltage on a plunger gate 
(a)
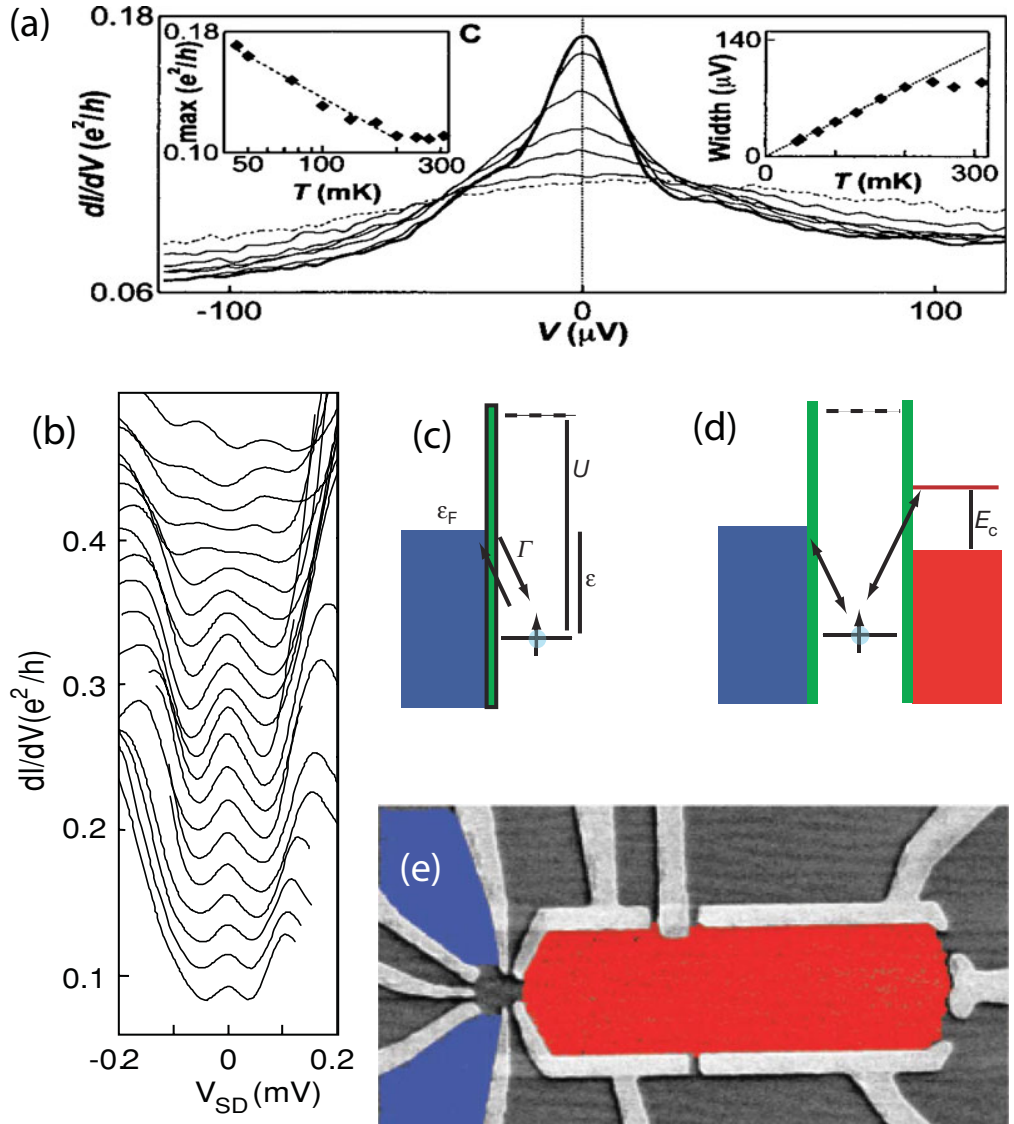

(d)
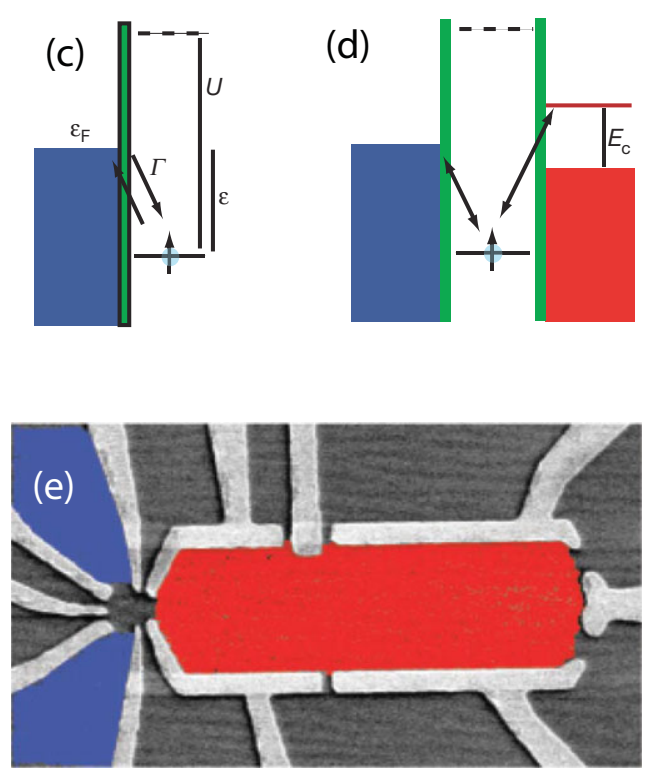

Figure 7 Kondo Physics in Quantum Dots. (a) Zerobias peak in the differential conductance for a single quantum dot with $\mathrm{S}=1 / 2$ (from Ref. [85]). [inset] Temperature dependence of the height and width of the peak, showing the Kondo character of the resonance. (b) In double dots, the interdot coupling leads to a splitting of the zero-bias peak (from Ref. [91]) (c) Principle of the single dot - single reservoir Kondo effect: exchange of electron with the reservoir by cotunneling. (d) Two-reservoirs Kondo effect: a different charging energy $E_{c}$ of the red reservoir prevents exchange of electrons between reservoirs and allows simulating the 2-channel Kondo effect. (e) SEM image of a 2-channel Kondo dot. [90] (f-g) Scaling of the differential conductance for 2-channel (from Ref. [90]) (2CK, f) and 1-channel (1CK, g) Kondo effect, showing the $2 \mathrm{CK}$ character of the zero-bias anomaly in a carefully tuned device.

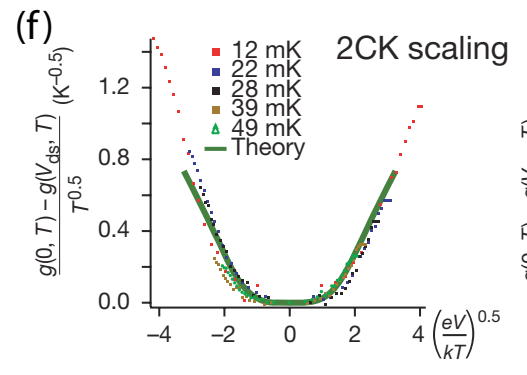

(f)

capacitively coupled to the finite reservoirs, the authors could tune the strength of its Kondo coupling with the quantum dot, leaving the coupling to the infinite reservoir unchanged. Measuring the conductance between the two (blue colored) leads of the infinite reservoir, they observed a Kondo peak when the localized electron couples most strongly with the infinite reservoir, a Kondo dip when the localized electron couples most strongly with the finite reservoir, both resulting from single-channel Kondo effects. Between these two situations, the scaling of the conductance with bias and temperature (see Fig. 7f-g) showed a two-channel Kondo effect, the signature of dot-mediated spin correlations in the two reservoirs.
The two-impurities Anderson model [92] can be simulated in double quantum dot. In this model, the spins on the two impurities can interact via Heisenberg exchange (with an exchange energy $J$ ) and couple to electron reservoirs via Kondo coupling (with a Kondo temperature $T_{K}$ ). A quantum phase transition exists as a function of the relative strength of these two couplings. At zero temperature, a quantum critical point is found at $J=J_{c}=2.2 T_{K}$. For $J<J_{c}$, the spin in each of the impurities is screened by the electrons in the reservoirs, and no inter-impurity correlations exist. For $J>J_{c}$, the interimpurity coupling is strong enough to form a spin singlet in the double quantum dot: the ground state is therefore a superposition of the two local Kondo states, which form 
bonding and antibonding states. For quantum dots, this effect was predicted to result in the splitting of the Kondo peaks for large interdot tunnel coupling [93-95]. Jeong et al. [30] observed these splitted Kondo peaks in a serial double quantum dot. However, they could not achieve a high enough Kondo temperature to reach the quantum phase transition, which was later observed [91] for parallel coupled quantum dots.

Future experiments could study Kondo physics with three dots, either in a in-line geometry [96], or in a triangular geometry [97-100]. The triangular geometry is especially interesting as it adds Aharonov-Bohm physics, and allows studying its interplay with the Kondo and Heisenberg coupling. Experimental developments are very encouraging. Indeed, a triangular triple quantum dot with tunnel coupling between every pair of dots has been realized recently [73]. In this paper, the authors showed that the capacitive coupling between different dot pairs could simulate a Ising hamiltonian acting on the charge degrees of freedom. Taking advantage of the triangular geometry and the associated frustration of the Ising couplings, they created a sixfold degenerate ground state, similar to the ground state of a frustrated spin system [7]). It is interesting to note that while spin-spin coupling in quantum dots follows the Heisenberg hamiltonian (see equation (4), charge-charge interaction allow simulating the Ising model. Other proposals exit for capacitively coupled quadruple dots, similar to those used for double singlet-triplet qubits [48], to be used to study in details the competition between Kondo, Heisenberg, and Ising coupling [101].

As we have seen, quantum dot systems offer a very interesting platform for quantum simulations. Not only can they be operated as qubits, but the versatility of their design offers the possibility to perform analog quantum simulations for Hubbard, Ising, Heisenberg or Kondo hamiltonians in different situations. Moreover, the efficient control of the quantum dot properties through gate voltages offer a very practical way to tune the parameters of the Hamiltonian and explore various physical regimes.

\section{Large dot arrays}

\subsection{Motivation}

The real motivation for quantum simulations is the possibility to access physics that cannot be explored otherwise. Whereas small systems can still be simulated on classical computers, this is no longer true for larger systems, as the time and effort required for such a simula- tion increases exponentially with the number of degrees of freedom. In this section, we discuss the prospects of realizing large-scale quantum simulation of the Hubbard model using quantum dot arrays. This would allow simulating the foundations of condensed matter physics in artificial electronic systems.

Instead of using a bottom-up approach and trying to control individually an increasing number of dots, we here present a top-down approach that directly defines a large array of dots, with global (non site-selective) control knobs. A large periodically spaced array of sites can be created with a grid-shaped gate (see figure $8 b$ ), which defines a periodic modulation of the electrostatic potential within the 2DEG. This establishes an electronic lattice, similar to the optical lattices used for cold atoms [2-4].

A major factor in experiments on large quantum dot arrays is disorder in the potential landscape due to charged impurities in the substrate [102]. In the few-dot experiments discussed in section 2 , the disorder could be compensated for by adjusting the gate voltages controlling the individual dots. When a large array is created via a single gate shaped in the form of a grid, such local compensation is no longer possible. In section 3.2, we discuss initial experiments on such systems aimed at establishing that the periodic potential induced by the grid gate can exceed the spatial variations in the potential from disorder. In section 3.3, we present ideas and experiments of the last few years directed at probing the influence of electron-electron interactions.

\subsection{Periodicity}

The idea of laterally modulating the electrostatic potential in a periodic way is not new: first experiments using a grid-shaped gate were done in 1989 [104]. In the following years, many groups have studied the magnetoresistance of periodically modulated systems, called "lateral superlattices" or "antidot lattices". These antidot lattices can be seen as a lattice of quantum dots with an extremely high coupling strength: instead of being localized in dots, electrons are localized everywhere besides at the antidots. This can be achieved either using a grid-shaped gate or by etching physical holes in the heterostructure.

Magnetoresistance measurements provide a sensitive probe for observing the formation of an artificial band structure in a $2 \mathrm{D}$ electron system. The electronic states in the conduction band obey the Bloch phase relation $|\psi(r+R)\rangle=e^{i k R}|\psi(r)\rangle$, which needs to match the phase acquired by electrons moving in a magnetic field. This 
a

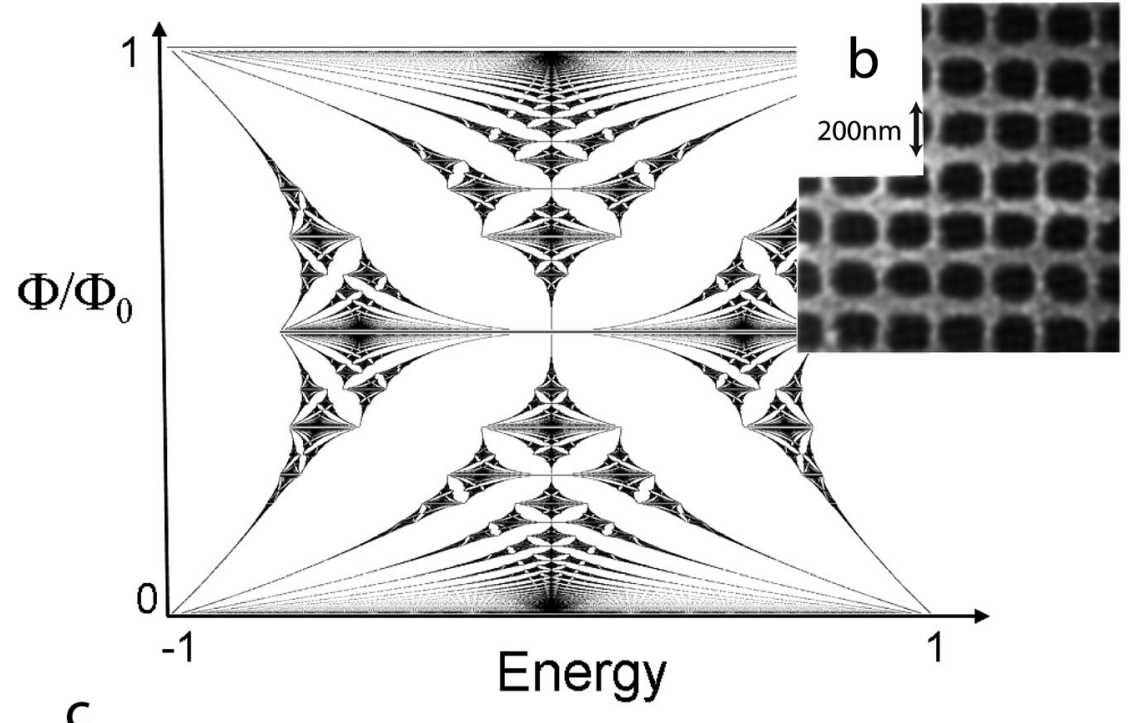

C

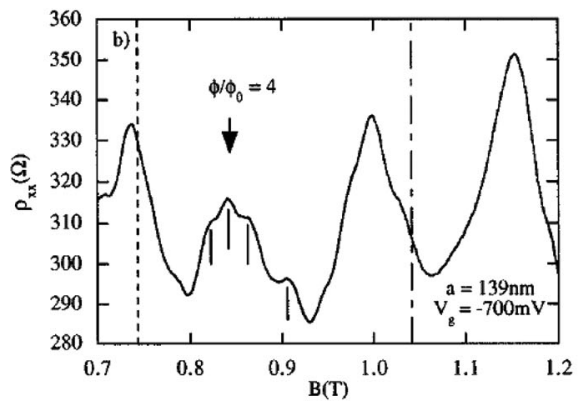

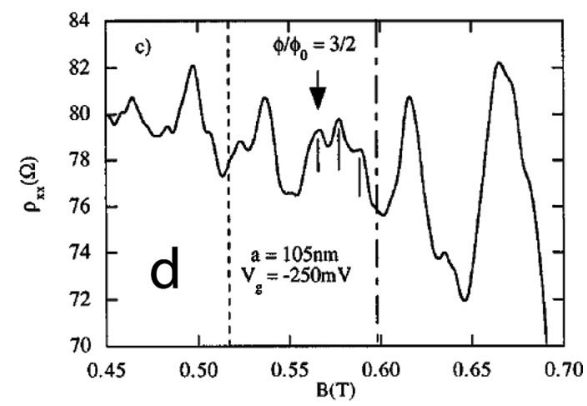

Figure 8 Magnetotransport in antidot arrays. (a) Calculated Hofstadter's butterfly spectrum, showing a fractal set of states. (from Ref. [103]) (b) SEM image of a gated antidot lattice (from Ref. [104]). (c-d) Splitted commensurability peaks observed in magnetotransport (from Ref. [105]). interplay between magnetic field and periodicity results in a fractal spectrum that resembles a butterfly, known as Hofstadter's butterfly [103] (see Fig. 8a): when the ratio $\Phi / \Phi_{0}$ of the magnetic flux in one unit cell and the flux quantum $\Phi_{0}$ is a rational number $p / q$, the Bloch band splits in exactly $q$ subbands (see Fig. 8a). Here $\Phi_{0}=h / 2 e$, with $h$ Planck's constant.

For solid-state crystals, the period of the lattice $a \sim$ 1 Å makes Hofstadter's butterfly extremely difficult to observe, as gigantic magnetic fields would be needed to achieve a magnetic flux per unit cell comparable to the flux quantum. It was therefore proposed by Hofstadter to realize large-period superlattices having a period of the order of $a \sim 100 \mathrm{~nm}-1 \mu \mathrm{m}$ (Fig. 8b) to observe the effect under reasonable experimental conditions.

Many experiments aimed therefore at the study of periodic systems in a magnetic field. Peaks in the magnetoresistance were first observed when the cyclotron radius was commensurate with the periodicity of the antidot array [106] and were due to classical drift of the center of the cyclotron orbits. Later Schlösser [105], and Albrecht [107] observed the splitting of these peaks for rational values of $\phi / \phi_{0}$ (see Fig. 8c-d) that they in- terpreted as the precursors of the largest minigaps in the band structure. Albeit Hofstadter Butterfly effects have been very recently observed in graphene systems [108-110], the strong disorder of the heterostructures prevented so far a clear and convincing observation of the butterfly in GaAs systems.

Modifying the design of the antidot lattices allowed defining arrays of quantum dots. First, Dorn et al. [111], using local oxidation to modulate the potential and a top gate to modulate the electron density, created a device where they could tune the inter-site tunnel coupling both in the antidot lattice regime and in the quantum dot array regime. They observed both the commensurability magnetoresistance peaks in the former and Coulombblockade effects in the latter. More recently, Goswami et al. [112] built a double-gate system allowing to tune independently electrochemical potential and interdot tunnel coupling.

We emphasize that the flexibility given by nanofabrication techniques permits to create not only square lattices, but any type of lattice. For instance, Gibertini et al. [113] proposed to realize an hexagonal array of quantum dots to create artificial graphene and study Dirac 
fermions physics. Following this proposal, de Simoni et al. [114] created a 2D hexagonal lattice of etched quantum dots. They observed a conductor-insulator transition controlled by gate voltage, resulting from the vanishing of the interdot coupling upon increasing the dot confinement.

\subsection{Interactions}

The most interesting regime in the context of quantum simulation occurs when electron-electron interactions enter the picture. The band structure effects of the previous section concern in essence single-particle physics, but this is no longer the case when interactions are relevant. A proposal for simulating interaction effects using quantum dot arrays has been made recently by Byrnes [115], focusing on the Mott metal-insulator transition (MIT): in the large tunnel coupling limit $(t>U)$, electrons are delocalized over the whole array and ensure a large metallic conductivity, while for low tunnel coupling ( $t \ll U / 8)$, a gap opens at half-filling (1 electron per site) and splits the conducting band into two Mott-Hubbard subbands. This gap suppresses the double occupancies in the dots and halts transport by localizing the electrons. Byrnes calculated the voltage dependence of $U, t$ and the electrochemical potential for quantum dot systems, and showed that the MIT should be observable in realistic quantum dots arrays.

Also optical spectroscopy (inelastic light scattering) can be used to explore band structure and interaction effects. Initial measurements on hexagonal arrays of decoupled dots [116] showed an unusual resonance peak. The energy of this peak scaled with the magnetic field as $E \propto \sqrt{B}$. The authors claimed that this scaling was due to electron-electron interactions, and attributed the resonance to an excitation between Mott-Hubbard subbands. The interdot tunnel coupling in the array was negligible and the disorder was strong enough to induce variations of the charge number $N$ on different dots, which suggests electrostatic potential fluctuations of the order of the charging energy (typ. $1-5 \mathrm{meV}$ ).

Whereas both charge and spin ordering have been observed in few-dot systems, the strong disorder of the materials has so far strongly hindered their observation in large arrays of quantum dots. The typical electrostatic potential fluctuations mentioned above is of the order of the interaction energy, which makes it one of the dominant terms of the Hamiltonian. As we have seen, it can destroy the effects of periodicity and interactions, thereby destroying spin and charge ordering, or making
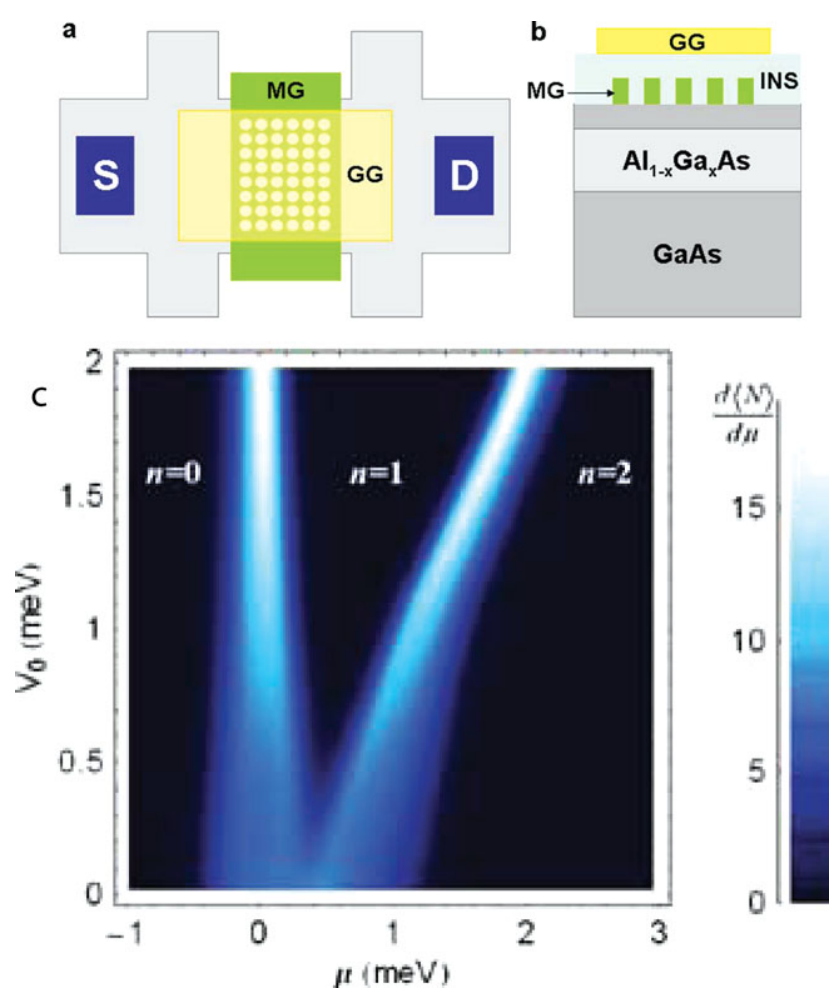

Figure 9 Simulating Hubbard physics in an array of quantum dots (from Ref. [115]). (a-b) Proposed transport measurement device: an array of quantum dots is defined by a mesh gate (MG), that controls the depth of the periodic potential, and a global gate (GG) that controls the electrochemical potential $\mu$. (c) Calculated density of states, as a function of the electrochemical potential and the potential $V_{0}$ of the mesh gate. One can see the splitting of the band in two Mott-Hubbard subbands.

the interpretation of experiments ambiguous. In the next section, we will describe new ideas to tackle this challenge and go towards clean simulations of the Hubbard model using quantum dot arrays.

\section{Overcoming disorder: towards quantum simulations in clean arrays}

Disorder adds to the Hubbard hamiltonian local fluctuations of the potential. Instead of the Hubbard model, the system is better described by the Anderson-Hubbard model:

$H=-t \sum_{i, j} c_{i}^{\dagger} c_{j}+U \sum_{i} n_{i, \uparrow} n_{i, \downarrow}+\sum_{i} \mu_{i} n_{i}$, 
(a)
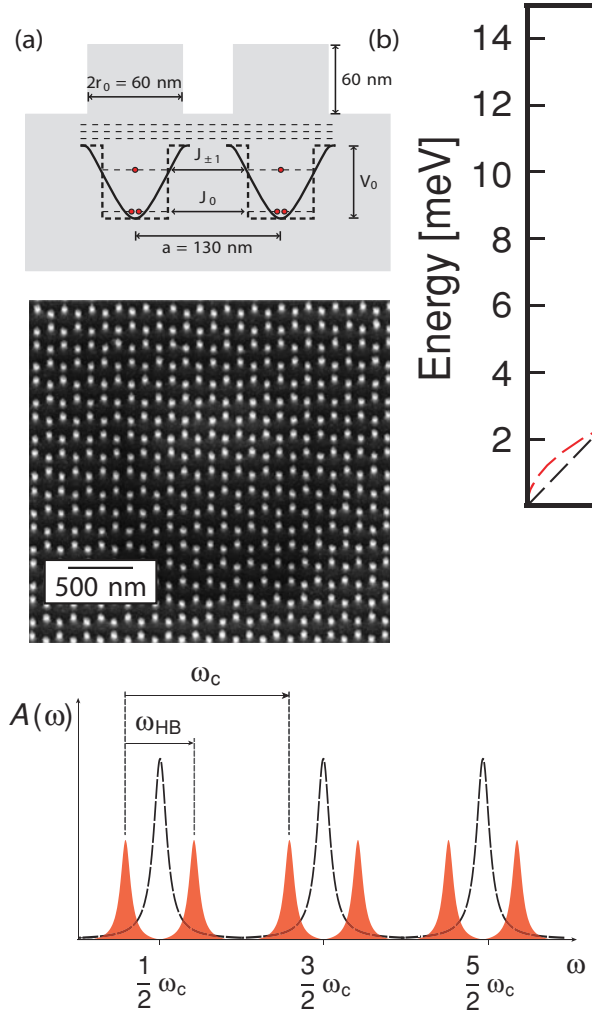
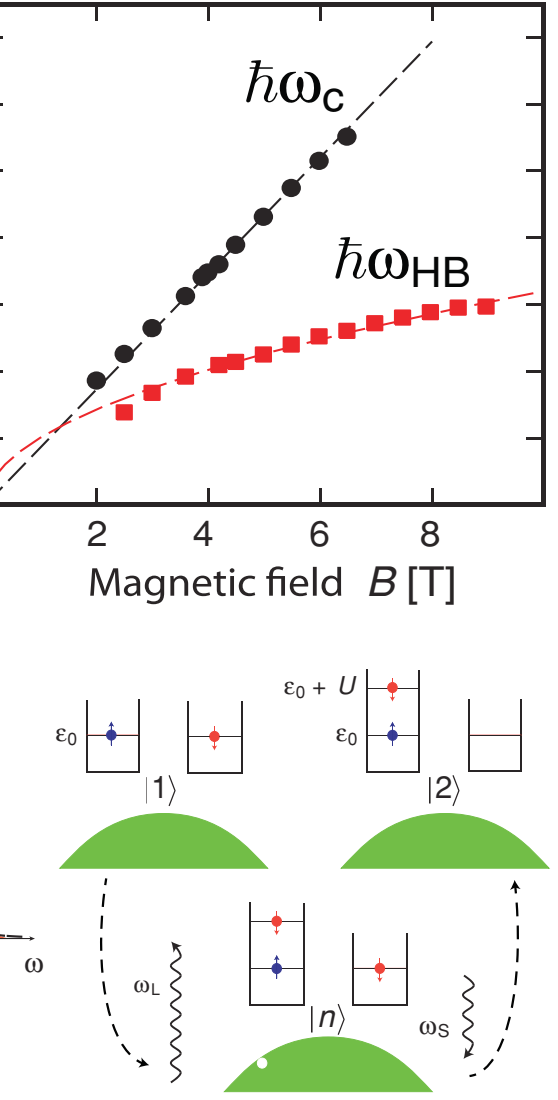

Figure 10 Artificial graphene experiments (from Ref. [116]). (a) Design of an etched heterostructure to create a periodic modulation of the potential on a hexagonal lattice. (b) Energy of the inelastic scattering peaks: one peak corresponds to excitations between Landau levels $\omega_{c} \propto B$ and one peak with $\omega_{H B} \propto \sqrt{B}$ is attributed to excitations between Mott-Hubbard subbands. (c) Energy level diagram showing the Landau level splitted in two subbands. (d) Proposed excitation scheme for the $\omega_{H B}$ peak: a photon with frequency $\omega_{L}$ excites an electron-hole pair. The hole recombines with an electron of one of the neighbouring dots, emitting a photon with frequency $\omega_{S}=\omega_{L}-\omega_{H B}$. where $\mu_{i}$ is now a randomly fluctuating variable. It is clear that the presence of this extra term can dramatically influence the behaviour of the system, especially if the order of magnitude of the variations of $\mu_{i}$ are large compared to the two other terms of the Hamiltonian, namely the tunnel coupling $t$ and the interaction energy $U$. As suggested by the site-to-site charge fluctuations observed by Singha et al. [116], potential fluctuations are of the order of $\Delta \mu \approx U$, in the meV range, consistently with early measurements of disorder in $2 \mathrm{D}$ electron systems [102].

Disorder also has a detrimental effect on transport measurements, as it induces Anderson Localization $[117,118]$. This disorder-induced localization of the electrons results in an insulating state, which prevents using conductance measurements to access Hubbard physics.

In this section, we propose new ideas to decrease the influence of disorder in quantum dot arrays that could allow the observation of Hubbard physics in these arrays. First, using undoped heterostructures built to screen disorder would strongly decreases the potential fluctuations. Second, capacitance spectroscopy methods offer access to the quantum state of electrons in a quantum well independently of the presence of Anderson Local- ization. This type of measurement is thereby much less sensitive to disorder.

\subsection{Reducing disorder}

Semiconductors always contain charged impurities. These can be desired, such as the dopants one introduces to form a 2DEG, or can be undesired bulk or surface impurities. The relatively large number of impurities all interact with the electrons in the 2DEG, which results in strong fluctuations of the electrostatic potential.

The dominant source of disorder for state-of-the-art samples come from the dopants. These are generally placed in a layer tens of nanometers above the 2DEG, and have a sheet concentration of the order of $10^{12} \mathrm{~cm}^{-2}$. The second most important are the undesired background (bulk) impurities, which can have a density as low as $1-5 \times 10^{13} \mathrm{~cm}^{-3}[119,120]$, and cause most of the disorder in undoped systems [121]. Surface charges can also induce disorder, and mainly influence shallow (30 - $40 \mathrm{~nm}$ deep) 2DEGs. As the surface charges can be efficiently screened by metallic gates [122] we will focus on the influence of doping and background impurities. 
Byrnes proposed to use undoped heterostructures [115], which would remove the dominant contribution to the disorder. We propose further to grow special heterostructures, containing a 3D gas of free electrons below the 2DEG, in order to screen the residual disorder originating from background impurities.

To estimate the efficiency of the screening for reducing the disorder, we performed the following calculation: first, we calculated the total potential created in the plane of the 2DEG by a single impurity. In cylindrical coordinates $(q, z)$, the Fourier transform of the potential due to a charge placed on $\left(r=0, z=z_{c}\right)$ is given by:

$$
\begin{aligned}
V_{t o t}\left(q, z, z_{0}\right)= & e U\left(q, z-z_{C}\right) \\
& +\int U\left(q, z-z_{0}\right) \rho_{\text {ind }}\left(q, z_{0}\right) d z_{0},
\end{aligned}
$$

where $U(q, z)$ is the Fourier transform of the singlecharge coulomb potential, and $\rho_{\text {ind }}$ is the charge density induced by the single charge. In the structures proposed below, this induced charge is non-zero only in the 2DEG or in screening layers. This charge density is related to the total potential. In the simple Thomas-Fermi approximation, we have:

$\rho_{\text {ind }}(q, z)=\chi(q, z) V_{\text {tot }}(q, z)$

where the susceptibility is given by $\chi_{3 D}=-e^{2} m^{*} k_{f} / \hbar^{2} \pi^{2}$ ( $\chi_{2 D}=-e^{2} m^{*} / \hbar^{2} \pi^{2}$ ) for a 3D (2D) gas of electrons. $e$ is the electron charge, $m^{*}$ is the effective electron mass and $k_{f}$ is the Fermi wavevector. We then obtain the integral equation

$$
\begin{aligned}
e_{\chi} U\left(q, z-z_{C}\right)= & \rho_{\text {ind }}(q, z) \\
& -\chi \int U\left(q, z-z_{0}\right) \rho_{\text {ind }}\left(q, z_{0}\right) d z_{0}
\end{aligned}
$$

Discretizing and solving this equation allows to calculate, for any scheme of screening, the potential in the plane of the 2DEG due to a single localized impurity.

To estimate the strength of the total disorder, we sum the contributions of a realistic number of background and doping impurities, whose position where randomly chosen (uniform 3D probability distribution for the background impurities, uniform 2D probability distribution and fixed depth for dopants) in a volume of $10 \times 10 \mu \mathrm{m} \times 200 \mathrm{~nm}$. We performed this estimation of the disorder for the following systems: sample 1 (Fig. 11a) has the typical parameters for gate defined quantum dots: it has a concentration of background impurities of $N_{b g}=10^{14} \mathrm{~cm}^{-3}$ and a sheet density of dopants of $N_{\text {dopants }}=10^{12} \mathrm{~cm}^{-2}$ in a layer placed $35 \mathrm{~nm}$ below the surface. In this sample, the 2DEG is placed $85 \mathrm{~nm}$ below the surface. Sample 2 (Fig. 11b) has a structure identical to sample 1 , but is undoped. The heterostructure of sample 3 is shown in Fig. 11c. It is undoped, has the 2DEG $70 \mathrm{~nm}$ below the surface, and a 3D gas of charges $15 \mathrm{~nm}$ below it.

Three questions need to be addressed: first, what is the reduction of the disorder obtained by using undoped structures (compare sample 1 and 2); second, what is the reduction of the disorder obtained by placing a screening layer below the 2DEG (compare sample 2 and 3); third, we need to evaluate the influence of disorder for the case where electrons in the 2DEG itself are no longer mobile due to the confinement in a quantum dot array. To do that, we compare the potential fluctuations with and without screening from the electrons in the 2DEG.

Figure 11d,e,f show the calculated potential for samples 1, 2 and 3, when the 2DEG does participate in the screening. One can see a strong reduction of the fluctuations between sample 1 and 2 and a smaller improvement between sample 2 and 3. Fig. 11g,h,i shows the calculated potential without screening from the 2DEG. One can see that the potential fluctuations in sample 2 have increased, while this effect is strongly reduced in sample 3.

To give a quantitative estimation of the strength of the disorder, we use the the cumulative probability distribution $C(V)=\int_{0}^{V} P\left(\delta_{V}\right) d \delta_{V}$ (Fig. 11(j)), where $P\left(\delta_{V}\right)$ is the probability to observe locally a potential fluctuation $\delta_{V}=$ $|V(x, y)-<V(x, y)>|$. For instance, $C(V)=90 \%$ means that in $90 \%$ of the cases, the local potential fluctuation is lower than $V$. A typical energy scale for the fluctuations $\Delta V$ is chosen as $C(V=\Delta V)=50 \%$. The doped samples have typical fluctuations $\Delta V=5.4 \mathrm{meV}$. This value is similar to other calculations [123] and is consistent with experimentally observed effects $[102,116]$. This value goes down to $\Delta V \approx 40 \mu \mathrm{eV}$ for sample 2 and $\Delta V \approx$ $10 \mu \mathrm{eV}$ for sample 3 . Without $2 \mathrm{D}$ screening, $\Delta V \approx 1 \mathrm{meV}$ for sample 2 , consistent with experiments in similar systems [124] and $\Delta V \approx 30 \mu \mathrm{eV}$ for sample 3 .

From these estimations, one can see that using undoped heterostructures with a screening layer below the 2DEG should strongly decrease the strength of the disorder to below tunneling energies in realistic dot arrays $(t \simeq 100 \mu \mathrm{eV})$. High quality heterostructures can show background impurity concentrations five times lower than the concentration used in the above estimates. Finally further improvements could come from quantum dots populated with more than one electron. Indeed, for few-electron quantum dots, deep shell electrons participate in the screening process and decrease the potential fluctuations seen by the last electron [125]. 
(a)

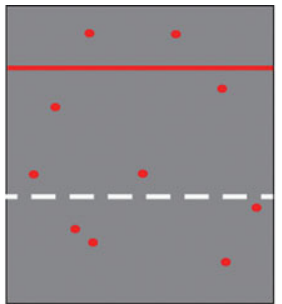

(d)

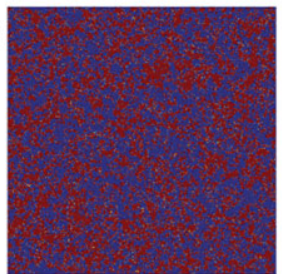

(g)

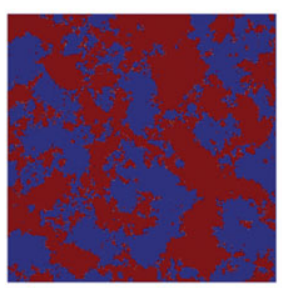

(b)

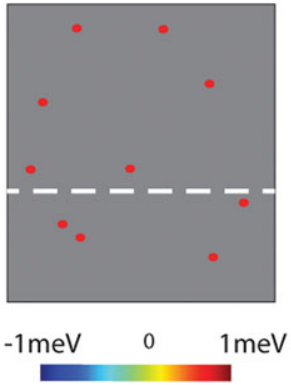

(c)

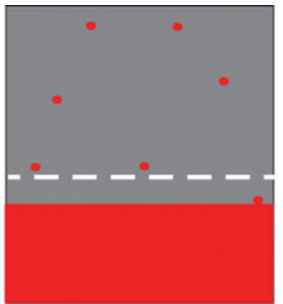

(e)

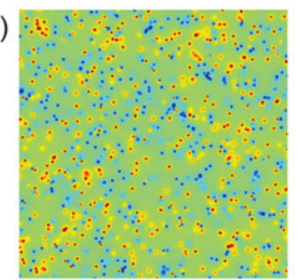

(h)

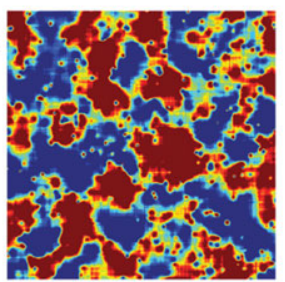

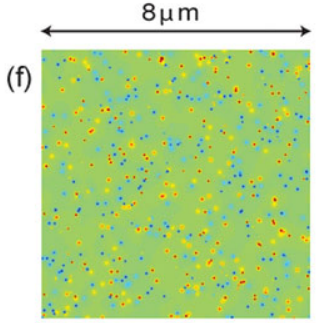

(i)

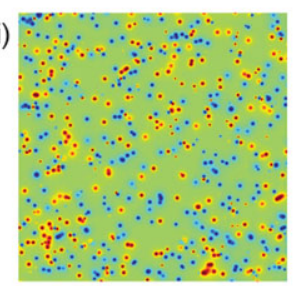

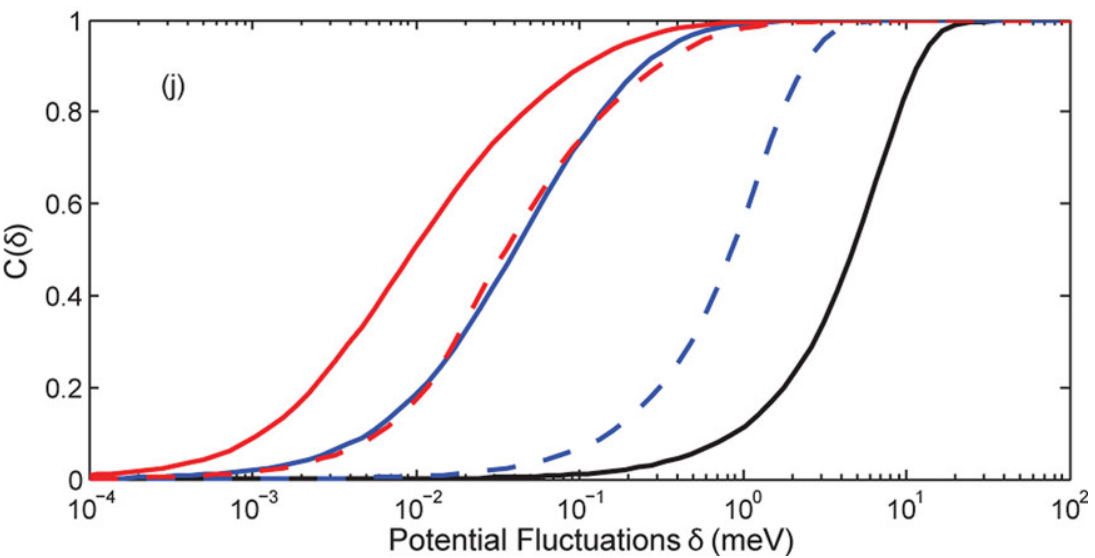

Figure 11 Influence of the screening scheme on the electrostatic potential fluctuations. (a,b,c) Schematic structure of samples 1,2 and 3. The white dashed line represents the $2 \mathrm{DEG}$ ( $86 \mathrm{~nm}$ below the surface for sample 1 and 2 , $70 \mathrm{~nm}$ for sample 3 ), the red line is the doping layer ( $30 \mathrm{~nm}$ below the surface). Red dots indicate undesired impurities, placed randomly in the sample. The red rectangle represents the 3D gas of charges. (d,e,f) Calculated potential fluctuations with 2D screening. (g,h,i) Calculated potential fluctuations without 2D screening. (j) Cumulative probability distribution $C\left(\delta_{V}\right)$. For the doped case (black line), one can see that the typical fluctuations are in the meV range. For sample 2 (blue line), this falls to some tens of $\mu \mathrm{eV}$. For sample 3 (red line), is goes down in the $\mu \mathrm{eV}$ range. If the 2DEG does not participate in the screening (dashed lines), the screening layer in sample 3 keeps the fluctuations low $(\Delta V \approx 10 \mu \mathrm{eV}$ red dashed line), while they strongly increase for sample 2 .

\subsection{An alternative to transport measurements}

For heterostructures with a screening layer, fabrication of devices for magnetotransport experiments are made difficult by the proximity of the 2DEG and the screening layer, and may require complex fabrication techniques [126] with relatively low yields. Moreover, as we have seen, due to their sensitivity to Anderson Localization [102], conductance measurements are not the ideal method to study the quantum state of electrons in an array of dots. A valuable alternative is to perform tunneling (capacitive) spectroscopy [127, 128].

For a thin $(\sim 10-15 \mathrm{~nm})$ AlGaAs barrier separating the 2DEG and the screening layer, tunneling of charges can occur between the two layers with frequencies tun- able by the barrier thickness $\left(v_{t} \approx 10-100 \mathrm{kHz}\right.$ is a useful range). Low-frequency $\left(v<v_{t}\right)$ measurements of the capacitance between the screening layer and the surface gates would then include a contribution from charges tunneling between the screening layer and the 2DEG (see figure 12c). As this contribution depends on the density of states in the 2DEG [127], it gives a convenient way to probe the electron quantum phases in the 2DEG. This offers an innovative option to probe the existence of a band structure and its distortion by magnetic fields (Hofstadter physics) or electron-electron interactions (Hubbard physics). Moreover, such a capacitive system can also be used for probing single-particle excitations (see Fig. 12d): fast voltage pulses on the back electrode allow charges to tunnel to excited states in the quantum well. 

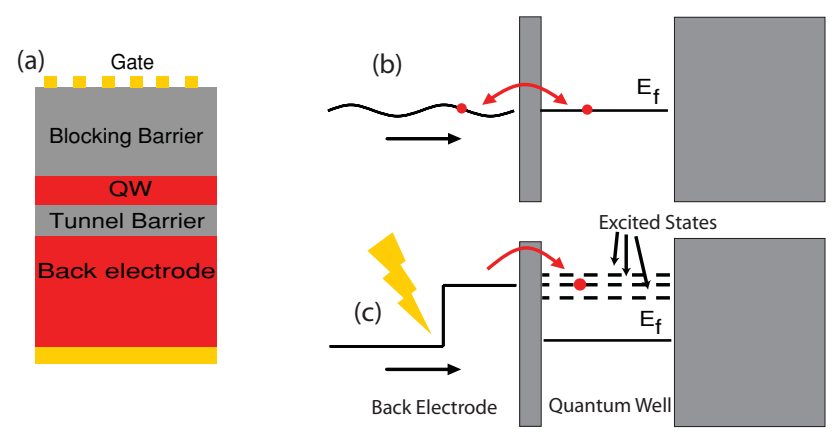

Figure 12 Capacitance Spectroscopy. (a) Scheme of the heterostructure. The barrier between the quantum well (OW) and the screening gas (back electrode) is sufficiently thin to allow tunneling of charges. (b) Equilibrium tunneling: a slow drive results in equilibrium tunneling of charges to the quantum well, which modifies the value of the capacitance. (c) Sending fast pulses to the back electrode drives the system out of equilibrium and induces tunneling to excited states in the quantum well. Both equilibrium and excited density of states can be accessed measuring the AC voltage on the gate.

The measurement of the charge transition rate as a function of the pulse amplitude allows to characterize the quasiparticle excitations of the 2DEG [128], which could open the way towards measurements of spectral-weighttransfer [129]. Moreover, the similarity of this technique to the tunneling spectroscopy used to observe the pseudogap phase in cuprate superconductors [130] opens interesting perspectives for studying the low-temperature Hubbard model in reduced-disorder quantum dots arrays.

\section{Outlook and conclusions}

Quantum dots systems have a very high potential for quantum simulations. They offer a large freedom in designing the geometry and a wide in-situ tunability of the parameters. For few-quantum dot systems, very insightful simulations have been performed, for instance studying Kondo physics in multi-channel or multiimpurity configurations. Moreover, the current technological progress open the way towards analog quantum simulations of frustration, spin chains, spin ladders, Nagaoka's ferromagnetism, and so forth. For large arrays of quantum dots, first experiments have been carried out, aimed at probing the effects of a periodic potential and interactions. Finally, new ideas could lead to strong reductions of the disorder and open the way towards quantum simulations of the low-temperature Hubbard model in large-scale systems.
Acknowledgements. We acknowledge useful discussions with Eugene Demler, Bernhard Wunsch, and Mark Rudner and the Delft spin qubit group, and financial support by the Netherlands Organization for Scientific Research (NWO), the Dutch Foundation for Fundamental Research on Matter (FOM), and the European Office of Aerospace Research and Development (EOARD).

Key words. Quantum simulation, quantum dots.

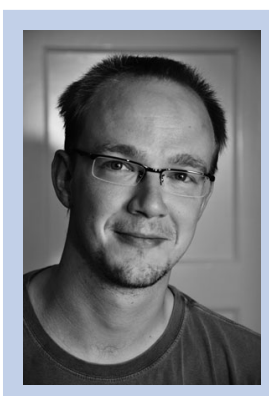

Pierre Barthelemy received his Ph.D. degree from the University of Florence in 2009 for a thesis on anomalous transport of light in disordered materials. He joined TU Delft as a post-doctoral fellow to work on electron transport in few quantum dot systems and received a VENI grant to develop large arrays of quantum dots for quantum simulations.

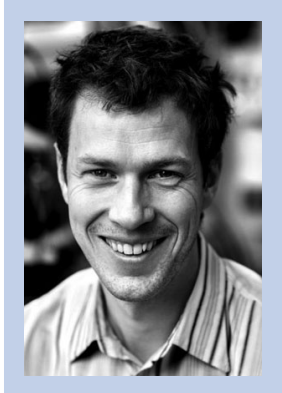

Lieven Vandersypen received his Ph.D. degree from Stanford University in 2001 for his work on NMR quantum computation. He is Antonie van Leeuwenhoek Professor at the Kavli Institute of Nanoscience at TU Delft (Netherlands). His research focus is on electron spin qubits in gated semiconductor quantum dots, and more recently on quantum simulation in this system.

\section{References}

[1] R. P. Feynman, International Journal of Theoretical Physics 21, 467 (1982).

[2] ColdAtomsAdP2013, Annalen Der Physik (2013).

[3] M. Greiner, O. Mandel, T. Esslinger, T. W. Hansch, and I. Bloch, Nature 415, 39 (2002).

[4] R. Jördens, N. Strohmaier, K. Günter, H. Moritz, and T. Esslinger, Nature 455, 204 (2008).

[5] G. B. Jo, Y. R. Lee, J. H. Choi, C. A. Christensen, T. H. Kim, J. H. Thywissen, D. E. Pritchard, and W. Ketterle, Science 325, 1521 (2009).

[6] S. Trotzky, Y. A. Chen, A. Flesch, I. P. McCulloch, U. Schollwöck, J. Eisert, and I. Bloch, Nature Physics 8, 3250 (2012).

[7] K. Kim, M. S. Chang, S. Korenblit, R. Islam, E. E. Edwards, J. K. Freericks, G. D. Lin, L. M. Duan, and C. Monroe, Nature 465, 590 (2010). 
[8] R. Gerritsma, G. Kirchmair, F. Zähringer, E. Solano, R. Blatt, and C. F. Roos, Nature 463, 68 (2010).

[9] TrappedIonsAdp2013, Annalen Der Physik (2013).

[10] S. Lloyd, Science 273, 1073 (1996).

[11] B. P. Lanyon, C. Hempel, D. Nigg, M. Muller, R. Gerristma, F. Zahringer, P. Schindler, J. Barreiro, M. Rambach, G. Kirchmair, P. Zoller, R. Blatt, and C. Roos, Science 334, 57 (2011).

[12] I. Bloch, J. Dalibard, and S. Nascimbène, Nature Physics 8, 267 (2012).

[13] R. Blatt, and C. F. Roos, Nature Physics 8, 277 (2012).

[14] A. Aspuru-Guzik, and P. Walther, Nature Physics 8, 285 (2012).

[15] PhotonicAdP2013, Annalen Der Physik (2013).

[16] A. A. Houck, H. E. Türeci, and J. Koch, Nature Physics 8, 292 (2012).

[17] SuperconductingAdP2013, Annalen Der Physik (2013).

[18] L. Kouwenhoven, D. Austing, and S. Tarucha, Reports on Progress in Physics 64, 701 (2001).

[19] W. G. Van Der Wiel, S. De Franceschi, J. M. Elzerman, T. Fujisawa, S. Tarucha, and L. P. Kouwenhoven, Reviews of Modern Physics 75, 1 (2003).

[20] R. Hanson, J. R. Petta, S. Tarucha, and L. M. K. Vandersypen, Reviews of Modern Physics 79, 1217 (2007)

[21] S. Trotzky, Y. a. Chen, U. Schnorrberger, P. Cheinet, and I. Bloch, Physical Review Letters 105, 265303 (2010).

[22] M. Imada, A. Fujimori, and Y. Tokura, Reviews of Modern Physics 70, 1039 (1998).

[23] P. A. Lee, and X. G. Wen, Reviews of Modern Physics 78, 17 (2006).

[24] M. Troyer, and U. J. Wiese, Physical Review Letters 94, 170201 (2005).

[25] T. Paiva, R. Scalettar, M. Randeria, and N. Trivedi, Physical Review Letters 104, 066406 (2010).

[26] F. R. Braakman, P. Barthelemy, C. Reichl, W. Wegscheider, and L. M. K. Vandersypen, Nature Nanotechnology 8, 432 (2013).

[27] F. A. Zwanenburg, A. S. Dzurak, A. Morello, M. Y. Simmons, L. C. L. Hollenberg, G. Klimeck, S. Rogge, S. N. Coppersmith, and M. A. Eriksson, Reviews of Modern Physics 85, 961 (2013).

[28] C. Fasth, A. Fuhrer, M. T. Björk, and L. Samuelson, Nano letters 5, 1487 (2005).

[29] F. Kuemmeth, S. Ilani, D. C. Ralph, and P. L. McEuen, Nature 452, 448 (2008).

[30] H. Jeong, A. M. Chang, and M. R. Melloch, Science 293, 2221 (2001).

[31] T. H. Oosterkamp, T. Fujisawa, W. G. Van Der Wiel, K. Ishibashi, R. V. Hijman, S. Tarucha, and L. P. Kouwenhoven, Nature 395, 873 (1998).

[32] A. Y. Smirnov, S. Savel, L. G. Mourokh, and F. Nori, Europhysics Letters (EPL) 80, 67008 (2007).

[33] M. Field, C. G. Smith, M. Pepper, D. A. Ritchie, J. E. F. Frost, G. A. C. Jones, and D. G. Hasko, Physical Review Letters 70, 1311 (1993).
[34] C. Barthel, M. Kjærgaard, J. Medford, M. Stopa, C. M. Marcus, M. P. Hanson, and A. C. Gossard, Physical Review B 81, 161308 (2010).

[35] K. C. Nowack, F. H. L. Koppens, Y. V. Nazarov, and L. M. K. Vandersypen, Science 318, 1430 (2007).

[36] J. R. Petta, A. Johnson, J. M. Taylor, E. A. Laird, A. Yacoby, M. D. Lukin, C. M. Marcus, M. P. Hanson, and A. C. Gossard, Science 309, 2180 (2005).

[37] I. Buluta, and F. Nori, Science 326, 108 (2009).

[38] M. A. Nielsen, and I. L. Chuang, Quantum Computation, and Quantum Information, 1 edition Cambridge University Press, Cambridge, (2004).

[39] T. Fujisawa, D. G. Austing, Y. Tokura, Y. Hirayama, and S. Tarucha, Nature 419, 278 (2002).

[40] T. Hayashi, T. Fujisawa, H. Cheong, Y. Jeong, and Y. Hirayama, Physical Review Letters 91, 226804 (2003).

[41] K. D. Petersson, J. R. Petta, H. Lu, and A. C. Gossard, Physical Review Letters 105, 246804 (2010).

[42] D. Loss, and D. P. DiVincenzo, Physical Review A 57, 120 (1998).

[43] F. H. L. Koppens, C. Buizert, K. J. Tielrooij, I. T. Vink, K. C. Nowack, T. Meunier, L. P. Kouwenhoven, and L. M. K. Vandersypen, Nature 442, 766 (2006).

[44] S. Nadj-Perge, S. M. Frolov, E. P. A. M. Bakkers, and L. P. Kouwenhoven, Nature 468, 1084 (2010).

[45] M. Pioro-Ladrière, T. Obata, Y. Tokura, Y. S. Shin, T. Kubo, K. Yoshida, T. Taniyama, and S. Tarucha, Nature Physics 4, 776 (2008).

[46] K. C. Nowack, M. Shafiei, M. Laforest, G. Prawiroatmodjo, L. Schreiber, C. Reichl, W. Wegscheider, and L. Vandersypen, Science 333, 1269 (2011).

[47] J. Levy, Physical Review Letters 89, 147902 (2002).

[48] M. D. Shulman, O. E. Dial, S. P. Harvey, H. Bluhm, V. Umansky, and A. Yacoby, Science 336, 202 (2012).

[49] D. P. DiVincenzo, D. Bacon, J. Kempe, G. Burkard, and K. B. Whaley, Nature 408, 339 (2000).

[50] E. A. Laird, J. M. Taylor, D. P. DiVincenzo, C. M. Marcus, M. P. Hanson, and A. C. Gossard, Physical Review B 82, 075403 (2010).

[51] J. Medford, J. Beil, J. M. Taylor, S. D. Bartlett, A. C. Doherty, E. I. Rashba, D. P. Divincenzo, H. Lu, A. C. Gossard, and C. M. Marcus, Arxiv cond-mat 1302.1933 (2013).

[52] J. Medford, J. Beil, J. M. Taylor, E. I. Rashba, H. Lu, A. C. Gossard, and C. M. Marcus, Physical Review Letters 111, 050501 (2013).

[53] Elzerman, Hanson, W. V. Beveren, Witkamp, L. P. Kouwenhoven, and L. M. K. Vandersypen, Nature 430, 431 (2004).

[54] K. Ono, D. Austing, Y. Tokura, and S. Tarucha, Science 297, 1313 (2002).

[55] C. Barthel, D. Reilly, C. Marcus, M. Hanson, and A. Gossard, Physical Review Letters 103, 160503 (2009).

[56] A. Khaetskii, and Y. Nazarov, Physical Review B 64, 125316 (2001).

[57] T. Meunier, I. Vink, L. van Beveren, K. J. Tielrooij, R. Hanson, F. Koppens, H. Tranitz, W. Wegscheider, L. 
Kouwenhoven, and L. Vandersypen, Physical Review Letters 98, 126601 (2007).

[58] S. Amasha, K. MacLean, I. Radu, D. Zumbühl, M. Kastner, M. Hanson, and A. Gossard, Physical Review Letters 100, 046803 (2008).

[59] C. B. Simmons, J. R. Prance, B. J. Van Bael, T. S. Koh, Z. Shi, D. E. Savage, M. G. Lagally, R. Joynt, M. Friesen, S. N. Coppersmith, and M. A. Eriksson, Physical Review Letters 106, 156804 (2011).

[60] V. N. Golovach, A. Khaetskii, and D. Loss, Physical Review Letters 93, 016601 (2004).

[61] E. A. Chekhovich, M. N. Makhonin, A. I. Tartakovskii, A. Yacoby, H. Bluhm, K. C. Nowack, and L. M. K. Vandersypen, Nature Materials 12, 494 (2013).

[62] B. Urbaszek, X. Marie, T. Amand, O. Krebs, P. Voisin, P. Maletinsky, A. Högele, and A. Imamoglu, Reviews of Modern Physics 85, 79 (2013).

[63] M. Gaudin, Journal de Physique 37, 1087 (1976).

[64] F. Koppens, K. Nowack, and L. Vandersypen, Physical Review Letters 100, 236802 (2008).

[65] H. Bluhm, S. Foletti, I. Neder, M. Rudner, D. Mahalu, V. Umansky, and A. Yacoby, Nature Physics 7, 109 (2010).

[66] A. Wild, J. Kierig, J. Sailer, J. W. Ager, E. E. Haller, G. Abstreiter, S. Ludwig, and D. Bougeard, Applied Physics Letters 100, 143110 (2012).

[67] B. M. Maune, M. G. Borselli, B. Huang, T. D. Ladd, P. W. Deelman, K. S. Holabird, A. A. Kiselev, I. AlvaradoRodriguez, R. S. Ross, A. E. Schmitz, M. Sokolich, C. A. Watson, M. F. Gyure, and A. T. Hunter, Nature 481, 344 (2012).

[68] J. R. Prance, Z. Shi, C. B. Simmons, D. E. Savage, M. G. Lagally, L. R. Schreiber, L. M. K. Vandersypen, M. Friesen, R. Joynt, S. N. Coppersmith, and M. A. Eriksson, Physical Review Letters 108, 046808 (2012).

[69] A. C. Johnson, J. R. Petta, J. M. Taylor, A. Yacoby, M. D. Lukin, C. M. Marcus, M. P. Hanson, and A. C. Gossard, Nature 435, 925 (2005).

[70] H. Bluhm, S. Foletti, D. Mahalu, V. Umansky, and A. Yacoby, Physical Review Letters 105, 216803 (2010).

[71] S. Yang, X. Wang, and S. Das Sarma, Physical Review B 83, 161301 (2011).

[72] M. Busl, G. Granger, L. Gaudreau, R. Sánchez, A. Kam, M. Pioro-Ladrière, S. A. Studenikin, P. Zawadzki, Z. R. Wasilewski, A. S. Sachrajda, and G. Platero, Nature nanotechnology 8, 261 (2013).

[73] M. Seo, H. K. Choi, S. Y. Lee, N. Kim, Y. Chung, H. S. Sim, V. Umansky, and D. Mahalu, Physical Review Letters 110, 046803 (2013).

[74] R. Thalineau, S. Hermelin, A. D. Wieck, C. Bauerle, L. Saminadayar, and T. Meunier, Applied Physics Letters 101, 103102 (2012).

[75] V. V. Deshpande, M. Bockrath, L. I. Glazman, and A. Yacoby, Nature 464, 209 (2010).

[76] E. Dagotto, T. M. Rice, and H. J. S. Si, Science 271, 618 (1996).

[77] E. Dagotto, J. Riera, and D. Scalapino, Physical Review B 45, 5744 (1992).
[78] Y. Nagaoka, Physical Review 147, 3925 (1966).

[79] D. C. Mattis, International Journal of Nanoscience 2 , 165 (2003).

[80] J. von Stecher, E. Demler, M. D. Lukin, and A. M. Rey, New Journal of Physics 12, 055009 (2010).

[81] J. Kondo, Progress of Theoretical Physics 32, 37-49 (1964).

[82] P. Anderson, Physical Review 124, 41 (1961).

[83] M. Pustilnik, and L. Glazman, Journal of Physics: Condensed Matter 16, R513 (2004).

[84] D. Goldhaber-Gordon, H. Shtrikman, D. Mahalu, D. Abush-Magder, U. Meirav, and M. A. Kastner, Nature 391, 156 (1998).

[85] S. Cronenwett, T. Oosterkamp, and L. Kouwenhoven, Science 281, 540 (1998).

[86] S. Sasaki, S. De Franceschi, J. Elzerman, W. G. van der Wiel, M. Eto, S. Tarucha, and L. Kouwenhoven, Nature 405, 764 (2000).

[87] D. Cox, Physical Review Letters 59, 1240 (1987).

[88] C. L. Seaman, M. B. Maple, B. W. Lee, S. Ghamaty, M. Torikachvili, J. S. Kang, L. Z. Liu, J. W. Allen, and D. L. Cox, Physical Review Letters 67, 2882 (1991).

[89] T. Cichorek, A. Sanchez, P. Gegenwart, F. Weickert, A. Wojakowski, Z. Henkie, G. Auffermann, S. Paschen, R. Kniep, and F. Steglich, Physical Review Letters 94, 236603 (2005).

[90] R. M. Potok, I. G. Rau, H. Shtrikman, Y. Oreg, and D. Goldhaber-Gordon, Nature 446, 167 (2007).

[91] J. Chen, A. Chang, and M. Melloch, Physical Review Letters 92, 176801 (2004).

[92] B. A. Jones, C. M. Varma, and J. W. Wilkins, Physical Review Letters 61, 125 (1988).

[93] A. Georges, and Y. Meir, Physical Review Letters 82, 3508 (1999).

[94] R. Aguado, and D. Langreth, Physical review letters 85, 1946 (2000).

[95] R. López, R. Aguado, and G. Platero, Physical Review Letters 89, 136802 (2002).

[96] R. Žitko, and J. Bonča, Physical Review Letters 98, 047203 (2007).

[97] R. Sakano, and N. Kawakami, Physical Review B 72, 085303 (2005).

[98] T. Kuzmenko, K. Kikoin, and Y. Avishai, Physical Review Letters 96, 046601 (2006).

[99] R. Žitko, and J. Bonča, Physical Review B 77, 245112 (2008).

[100] A. Mitchell, T. Jarrold, and D. Logan, Physical Review B 79, 085124 (2009).

[101] D. E. Liu, S. Chandrasekharan, and H. U. Baranger, Physical Review Letters 105, 256801 (2010).

[102] S. Ilani, A. Yacoby, D. Mahalu, and H. Shtrikman, Science 292, 1354 (2001).

[103] D. Hofstadter, Physical Review B 14, 2239 (1976).

[104] K. Ismail, W. Chu, A. Yen, D. A. Antoniadis, and H. I. Smith, Applied Physics Letters 54, 460 (1989).

[105] T. Schlosser, K. Ensslin, J. Kotthaus, and M. Holland, Europhysics Letters (EPL) 33, 683 (1996).

[106] K. Ensslin, and P. Petrov, Physical Review B 41, 12307 (1990). 
[107] C. Albrecht, J. H. Smet, K. V. Klitzing, D. Weiss, V. Umansky, and H. Schweizer, Physical Review Letters 86, 147 (2001).

[108] B. Hunt, J. D. Sanchez-Yamagishi, A. F. Young, M. Yankowitz, B. J. LeRoy, K. Watanabe, T. Taniguchi, P. Moon, M. Koshino, P. Jarillo-Herrero, and R. C. Ashoori, Science 340, 1427 (2013).

[109] L. A. Ponomarenko, R. V. Gorbachev, G. L. Yu, D. C. Elias, R. Jalil, A. A. Patel, A. Mishchenko, A. S. Mayorov, C. R. Woods, J. R. Wallbank, M. MuchaKruczynski, B. A. Piot, M. Potemski, I. V. Grigorieva, K. S. Novoselov, F. Guinea, V. I. Fal'ko, and A. K. Geim, Nature 497, 594 (2013).

[110] C. R. Dean, L. Wang, P. Maher, C. Forsythe, F. Ghahari, Y. Gao, J. Katoch, M. Ishigami, P. Moon, M. Koshino, T. Taniguchi, K. Watanabe, K. L. Shepard, J. Hone, and P. Kim, Nature 497, 598 (2013).

[111] A. Dorn, T. Ihn, K. Ensslin, W. Wegscheider, and M. Bichler, Physical Review B 70, 205306 (2004).

[112] S. Goswami, M. A. Aamir, C. Siegert, M. Pepper, I. Farrer, D. A. Ritchie, and A. Ghosh, Physical Review B 85, 075427 (2012).

[113] M. Gibertini, A. Singha, V. Pellegrini, M. Polini, G. Vignale, A. Pinczuk, L. Pfeiffer, and K. West, Physical Review B 79(24), 241406 (2009).

[114] G. de Simoni, A. Singha, M. Gibertini, B. Karmakar, and M. Polini, Applied Physics Letters 97, 132113 (2010).

[115] T. Byrnes, N. Kim, K. Kusudo, and Y. Yamamoto, Physical Review B 78, 075320 (2008).

[116] A. Singha, M. Gibertini, B. Karmakar, S. Yuan, M. Polini, G. Vignale, M. Katsnelson, A. Pinczuk, L. Pfeiffer, K. W. West, and V. Pellegrini, Science 332, 1176 (2011).

[117] P. W. Anderson, Physical Review 109, 1492 (1958).
[118] E. Abrahams, P. W. Anderson, D. C. Licciardello, and T. V. Ramakrishnan, Physical Review Letters 42, 673 (1979).

[119] Y. Hirayama, K. Muraki, and T. Saku, Applied Physics Letters 72, 1745 (1998).

[120] V. Umansky, M. Heiblum, Y. Levinson, J. Smet, J. Nübler, and M. Dolev, Journal of Crystal Growth 311, 1658 (2009).

[121] S. Sarkozy, K. Das Gupta, C. Siegert, A. Ghosh, M. Pepper, I. Farrer, H. E. Beere, D. A. Ritchie, G. A. C. Jones, and K. D. Gupta, Applied Physics Letters 94, 172105 (2009).

[122] D. A. Kozlov, Z. D. Kvon, A. K. Kalagin, and A. I. Toropov, Semiconductors 41, 180 (2007).

[123] C. Zhou, M. Berciu, and R. Bhatt, Physical Review B 71, 125310 (2005).

[124] S. Ilani, J. Martin, E. Teitelbaum, J. H. Smet, D. Mahalu, V. Umansky, and A. Yacoby, Nature 427, 328 (2004).

[125] E. Barnes, J. P. Kestner, N. T. T. Nguyen, and S. D. Sarma, Physical Review B 84, 235309 (2011).

[126] K. Das Gupta, A. F. Croxall, J. Waldie, C. A. Nicoll, H. E. Beere, I. Farrer, D. A. Ritchie, and M. Pepper, Advances in Condensed Matter Physics 2011, 727958 (2011).

[127] R. Ashoori, J. Lebens, N. Bigelow, and R. Silsbee, Physical Review B 48, 4616 (1993).

[128] O. E. Dial, R. C. Ashoori, L. N. Pfeiffer, and K. W. West, Nature 448, 176 (2007).

[129] P. Phillips, Reviews of Modern Physics 82, 1719 (2010).

[130] C. Renner, B. Revaz, J. Y. Genoud, K. Kadowaki, and O. Fischer, Physical Review Letters 80, 149 (1998). 